Article

\title{
Poly(2-oxazoline)-Based Amphiphilic Gradient Copolymers as Nanocarriers for Losartan: Insights into Drug-Polymer Interactions
}

\author{
Angeliki Chroni ${ }^{1}$, Thomas Mavromoustakos ${ }^{2}$ (D) and Stergios Pispas ${ }^{1, *(\mathbb{D})}$ \\ 1 Theoretical and Physical Chemistry Institute, National Hellenic Research Foundation, 48 Vassileos \\ Constantinou Avenue, 11635 Athens, Greece; angelikechrone@gmail.com \\ 2 Organic Chemistry Laboratory, Department of Chemistry, National and Kapodistrian University of Athens, \\ Panepistimioupolis, 15771 Zografou, Greece; tmavrom@chem.uoa.gr \\ * Correspondence: pispas@eie.gr; Tel.: +30-210-727-3824
}

Citation: Chroni, A.;

Mavromoustakos, T.; Pispas, S.

Poly(2-oxazoline)-Based Amphiphilic

Gradient Copolymers as

Nanocarriers for Losartan: Insights into Drug-Polymer Interactions. Macromol 2021, 1, 177-200. https:// doi.org/10.3390/macromol1030014

Academic Editor: Ana

María Díez-Pascual

Received: 15 June 2021

Accepted: 28 June 2021

Published: 1 July 2021

Publisher's Note: MDPI stays neutral with regard to jurisdictional claims in published maps and institutional affiliations.

Copyright: (C) 2021 by the authors Licensee MDPI, Basel, Switzerland. This article is an open access article distributed under the terms and conditions of the Creative Commons Attribution (CC BY) license (https:// creativecommons.org/licenses/by/ $4.0 /)$.

\begin{abstract}
The current study is focused on the development of highly stable drug nanocarriers by encapsulating losartan potassium (LSR) into an amphiphilic biocompatible poly(2-methyl-2oxazoline)-grad-poly(2-phenyl-2-oxazoline) (PMeOxz72-grad-PPhOxz 28 ) gradient copolymer (GC). Based on dynamic light scattering (DLS), the PMeOxz72-grad-PPhOxz 28 (where the subscripts denote $\%$ wt composition of the components) GC formed micelles and aggregates of $13 \mathrm{~nm}$ and $96 \mathrm{~nm}$ in aqueous milieu. The presence of hydrophobic LSR molecules altered the structural characteristics of the GC, modulating the organization of the polymeric components and revealing the formation of hyper micellar nanostructures in addition to micelles. The 2D-NOESY experiments evidenced intermolecular interactions between the phenyl ring of LSR with the phenyl group of PPhOxz and eminent correlations between the butyl chain of LSR with the phenyl group of PPhOxz and methylene group of PMeOxz, respectively. Additionally, NMR studies as a function of temperature demonstrated that the presence of hydrophilic PMeOxz segments in the gradient core of $\mathrm{PMeOxz}_{72}$ grad-PPhOxz 28 nanoassemblies induced an increased fluidity of the core matrix, especially upon heating, thus causing water penetration, resulting in increased proton mobility. Lastly, the ultrasound release profile of LSR signified that a great amount of the encapsulated LSR is tightly bound to the PMeOxz 72 -grad-PPhOxz 28 nanoassemblies.
\end{abstract}

Keywords: amphiphilic gradient copolymers; polymeric nanocarriers; drug delivery systems; thin film hydration method; drug encapsulation; drug release profile; drug-polymer intermolecular interactions

\section{Introduction}

The progress of drug delivery technology has created a rich landscape of enterprising and promising polymer-based candidates for the demanding treatment of critical diseases [1,2]. Amphiphilic block copolymers (AmBCs) are now ubiquitous in drug delivery formulations, endowing drugs with favorable properties [3], such as water solubilization, enhanced stability and systemic circulation, increased uptake rate of target cells/tissues, reduced enzyme degradation and controlled release of the drug [4,5].

Amphiphilic gradient or pseudo-diblock copolymers (AmGCs) have revitalized the research in biomedical and pharmaceutical science, due to their distinctive molecular chain structure. The monomer composition of AmGCs varies gradually along molecular chains, unlike AmBCs, which have an abrupt change in monomer composition [6]. Commonly, the gradient copolymers (GCs) are synthesized by either living cationic polymerization $[7,8]$ or controlled living radical polymerization such as nitroxide-mediated radical polymerization (NMP) [9-11], atom transfer radical polymerization (ATRP) [12,13] or reversible additionfragmentation chain transfer (RAFT) [14-16]. 
AmGCs self-assemble into various aggregates in solutions under appropriate conditions, often exhibiting different structures and structural transitions compared to block copolymers (BCs) due to their special monomer arrangement within the polymer chain [17]. The micellization behavior of GCs can be triggered by temperature [15,16], $\mathrm{pH}[10]$ or solvent composition changes [18], similar to BCs. The level of GCs' micellization is lower compared to BCs with the same composition, due to the shorter effective length of solvophobic blocks [19]. Specifically, some of the solvophilic units may be integrated in the core while some solvophobic units may be distributed in the corona. Thus, GCs usually possess greater solubility than the corresponding BCs $[18,20,21]$, slower micellization process [22] and a lower value of the aggregation number $[19,23]$, reflecting a smaller size according to DLS [24].

Poly(2-oxazoline)s (POx) have been devised by researchers as the next generation of polymer therapeutics, meeting the criteria of biocompatibility, high modulation of solubility, size variation, architecture and chemical functionality [25]. A broad range of POx structures with variable chemical and physical features is produced by living cationic ringopening polymerization (CROP) of 2-oxazolines. POx with methyl or ethyl side groups are nonionic, non-toxic water-soluble polymers exhibiting stealth properties, whereas side groups longer than propyl or aryl segments provide increased hydrophobicity to the polyoxazolines [26-29]. Numerous seminal research papers have reported the design of amphiphilic POx-based drug delivery systems, exploiting their hydrophobic core as a matrix for the confinement of curcumin (CUR) [4], indomethacin (IND) [30], paclitaxel (PTX) [31], doxorubicin (DOX) [32], dexamethasone (Dex) [33], cabazitaxel (CAB) [34], and etoposide + cisplatin [35], among others.

Hitherto, only a few studies have utilized POx as a matrix for the encapsulation and delivery of antihypertensive drugs [36-38]. Sartan medicines (losartan, valsartan, candesartan, irbesartan, olmesartan, telmisartan, eprosartan, azilsartan) comprise the most recent class of antihypertensive therapeutics acting on the renin-angiotensin system [39-42]. Sartans as therapeutics suffer from hydrophobicity. A process to increase their bioavailability could be their encapsulation into POx, which is a totally unexplored research path, to the best of our knowledge.

Nuclear magnetic resonance (NMR) spectroscopy is an alternative, not fully explored characterization technique to illuminate the design and drug encapsulation efficiency of polymeric nanocarriers, by thorough examination of the micelle structure and polymerdrug intermolecular interactions $[43,44]$. Our previous works underlined the importance of NMR spectroscopy, which was the instrument to prove that the level of interaction between the polymers and the drug influenced the stability, encapsulation efficiency (\%EE), and drug release kinetics of the studied systems [45,46].

In the present work, we developed a novel drug delivery system by encapsulating losartan potassium (LSR) into a biocompatible and non-toxic poly(2-methyl-2-oxazoline)grad-poly(2-phenyl-2-oxazoline) ( $\mathrm{PMeOxz}_{72}$-grad-PPhOxz 28 , where the subscripts denote wt.\% composition of the components) GC and examined the intramolecular and intermolecular interactions between the polymer and the drug using NMR spectroscopy. The $\mathrm{PMeOxz}_{72}$-grad-PPhOxz 28 nanoassemblies and LSR-loaded PMeOxz72-grad-PPhOxz 28 nanocarriers (20 wt. \% and $50 \mathrm{wt} . \%$ concentration of LSR in the mixture) were prepared using the thin film hydration method (TFHM). An extensive physicochemical characterization was conducted using dynamic and electrophoretic light scattering (DLS, ELS) and attenuated total reflection Fourier transform infrared (ATR-FTIR) spectroscopy to assess the size, the surface potential, and the structure of nanoassemblies and nanocarriers (referred in the text as $\mathrm{PMeOxz}_{72}$-grad-PPhOxz $28+20 \% \mathrm{LSR}$ and $\mathrm{PMeOxz}_{72}$-grad-PPhOxz $28+50 \%$ LSR nanocarriers). The nanoassemblies and nanocarriers were studied in fetal bovine serum (FBS) to gain insight into the protein-polymer and protein-nanocarriers interactions. The physical stability of the nanocarriers was examined over a certain period of time using DLS. The encapsulation of LSR into the PMeOxz 72 -grad-PPhOxz 28 nanoassemblies was investigated through ultraviolet-visible (UV-Vis), ATR-FTIR, and NMR measurements in 
aqueous solutions. Particularly, experiments on ${ }^{1} \mathrm{H}-\mathrm{NMR}$, correlated (2D COSY), Overhauser effect (2D NOESY) and diffusion-ordered (2D DOSY) spectroscopy were performed to explore the intramolecular interactions between the PMeOxz72-grad-PPhOxz 28 GC and LSR, and to discover the self-diffusion coefficients D of the nanocarriers. Moreover, temperature studies of $\mathrm{PMeOxz}_{72}$-grad-PPhOxz 28 GC were pursued in the range $25-80{ }^{\circ} \mathrm{C}$ using ${ }^{1} \mathrm{H}-\mathrm{NMR}$ spectroscopy to trace the mobility of protons located in the hydrophobic gradient micellar core. Lastly, the drug loading (DL\%) and \%EE indexes were calculated by means of UV-Vis spectroscopy, followed by the ultrasound-triggered release of LSR from the $\mathrm{PMeOxz}_{72}$-grad-PPhOxz 28 nanoassemblies.

\section{Materials and Methods}

\subsection{Materials}

The following materials were used in our study: Losartan potassium (LSR, 99.8\%, Rafarm, Athens, Greece), ethanol (EtOH, 99.9\%, Sigma-Alrdrich, Athens, Greece), water for injection (WFI, 99\%, Sigma-Alrdrich), phosphate-buffered saline tablets (PBS, 98\%, SigmaAlrdrich, Athens, Greece), deuterium oxide $\left(\mathrm{D}_{2} \mathrm{O}, 99 \%\right.$, Sigma-Alrdrich) and fetal bovine serum (FBS, Sigma-Alrdrich, Athens, Greece). The synthesis of PMeOxz-grad-PPhOxz GC was performed using 2-methyl-2-oxazoline (MeOxz, 98\%, Sigma-Alrdrich, Athens, Greece) and 2-phenyl-2-oxazoline (PhOxz, 99\%, Sigma-Alrdrich, Athens, Greece) as the monomers and methyl p-toluenesulfonate (C8H10O3S 98\%, Sigma-Alrdrich, Athens, Greece) as the initiator.

\subsection{Preparation of Self-Assembled $P M e O x z_{72}$-Grad-PPhOxz 28 GC}

The synthesis of $\mathrm{PMeOxz}_{72}$-grad-PPhOxz 28 GC was performed by cationic polymerization and it has been described in detail in a previous work [30]. Due to the amphiphilic character and the gradient molecular structure of the $\mathrm{PMeOxz}_{72}$-grad-PPhOxz 28 copolymer, its solution behavior was studied in aqueous solutions at a polymer concentration of $\mathrm{C}=$ $10^{-3} \mathrm{~g} / \mathrm{mL}$ using the TFHM. Specifically, $5 \mathrm{mg}$ of the GC were firstly dissolved in $\mathrm{EtOH}$ (stock solution) and allowed to stand overnight for polymer dissolution at the molecular level. After $24 \mathrm{~h}$, the copolymer stock solution was transferred into a flask and placed in a rotary evaporator for the efficient evaporation of $\mathrm{EtOH}$ until a thin film of each polymer was formed around the inner part of the flask. When the thin film was formed, $5 \mathrm{~mL}$ filtered WFI was added to the flask and stirred until the entire thin film was dissolved. The concentration of the aqueous stock solution was $10^{-3} \mathrm{~g} / \mathrm{mol}$.

\subsection{Preparation of $L S R$-Loaded $P M e O x z_{72}-G r a d-P P h O x z_{28}$ Nanocarriers}

The PMeOxz 72 -grad-PPhOxz 28 + 20\% LSR and PMeOxz 72 -grad-PPhOxz 28 + 50\% LSR nanocarriers were also prepared using the TFHM. In particular, an appropriate amount of LSR was dissolved in EtOH to prepare $20 \mathrm{wt} . \%$ and $50 \mathrm{wt} . \%$ concentration of LSR in the final copolymer/drug mixture. After $24 \mathrm{~h}$, the copolymer and LSR stock solutions were mixed in the appropriate amounts. Then, each copolymer-drug mixture was transferred into a flask and placed in a rotary evaporator for the efficient evaporation of EtOH until a thin film of each copolymer and LSR was formed around the inner walls of the flask. Finally, $5 \mathrm{~mL}$ filtered WFI was added to the flask and stirred until the entire thin film was dissolved. The concentrations of the aqueous stock solutions were $10^{-3} \mathrm{~g} / \mathrm{mol}$. All copolymer and drug-loaded solutions were filtered through $0.45 \mu \mathrm{m}$ pore size filters and allowed to stand overnight for equilibration before measurements.

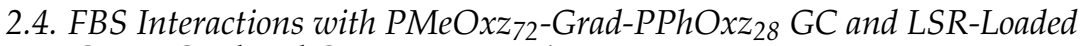 $\mathrm{PMeO} x z_{72}$-Grad-PPhOxz $z_{28}$ Nanocarriers}

The mixtures of $\mathrm{PMeOxz}_{72}$-grad-PPhOxz 28 GC and PMeOxz 72 -grad-PPhOxz $28+20 \%$ LSR and $\mathrm{PMeOxz}_{72}$-grad-PPhOxz 28 + 50\% LSR nanocarriers with clarified FBS were prepared in filtered PBS using two protocols based on different polymer dilution to FBS and different FBS:PBS ratios. The copolymer and copolymer-drug stock solutions were pre- 
pared with direct dissolution in PBS at a concentration of $3 \times 10^{-3} \mathrm{~g} / \mathrm{mL}$ for both protocols. According to the first protocol, $50 \mu \mathrm{L}$ of each diblock was mixed with (a) $3 \mathrm{~mL}$ FBS:PBS $(1 / 9 \mathrm{v} / \mathrm{v})$ ratio and (b) $3 \mathrm{~mL}$ FBS:PBS $(1 / 1 \mathrm{v} / \mathrm{v})$ ratio. The second protocol included the mixing of $100 \mu \mathrm{L}$ sample with (a) $3 \mathrm{~mL}$ FBS:PBS (1/9 v/v) ratio and (b) $3 \mathrm{~mL}$ FBS:PBS $(1 / 1 \mathrm{v} / \mathrm{v})$. All FBS-copolymer mixtures were filtered through $0.45 \mu \mathrm{m}$ pore size filters and allowed to stand $1 \mathrm{~h}$ for equilibration before DLS measurements.

\subsection{Drug Loading and Encapsulation Efficiency Calculations of LSR}

The percentage of LSR incorporated into PMeOxz 72 -grad-PPhOxz $28+20 \% \mathrm{LSR}$ and $\mathrm{PMeOxz}_{72}$-grad-PPhOxz 28 + 50\% LSR nanocarriers was estimated by UV-Vis (Perkin-Elmer, Lambda 19 spectrophotometer, Waltham, MA, USA) spectroscopy.

$\% \mathrm{DL}$ is the amount of drug loaded per unit weight of the micelle/nanoparticle, and is calculated by the amount of total entrapped drug divided by the total micelle/nanoparticle weight. \%EE is the percentage of drug that is successfully entrapped into the micelle/ nanoparticle and is calculated by the total encapsulated drug divided by the total drug added. \%DL and \%EE indexes were calculated using the following Equations:

$$
\begin{gathered}
\% \mathrm{DL}=[\text { encapsulated drug } / \text { total nanoassemblies weight }(\text { initial })] \times 100 \\
\% \mathrm{EE}=[\text { encapsulated drug } / \text { total drug added (initial) }] \times 100
\end{gathered}
$$

\subsection{Ultrasound Release Studies}

The release of LSR from $\mathrm{PMeOxz}_{72}$-grad-PPhOxz 28 nanoassemblies was studied in WFI in an ultrasonic bath sonicator for approximately five hours. Particularly, $5 \mathrm{~mL}$ of $\mathrm{PMeOxz}_{72}$-grad-PPhOxz 28 +50\% LSR nanocarriers were added in a dialysis bag of $3.5 \mathrm{kDa}$ MWDO. Afterwards, the dialysis bag was inserted into $100 \mathrm{~mL}$ filtered WFI and placed in a SOLTEC, SONICA 3300ETH-S3 ultrasonic bath. Aliquots of the samples were taken from the external solution at specific time intervals and each time the aqueous solution was restored to its initial volume, so that the reservoir conditions remained constant. The amount of LSR released at different time intervals, up to $5 \mathrm{~h}$, was determined using UVVis (Perkin-Elmer, Lambda 19 spectrophotometer, Waltham, MA, USA) spectroscopy at $\lambda \max =225 \mathrm{~nm}$.

\subsection{NMR Samples Preparation}

For NMR studies of $\mathrm{PMeOxz}_{72}$-grad-PPhOxz 28 GC, the solution was prepared by direct dissolution of $2 \mathrm{mg}$ of the copolymer in $0.7 \mathrm{~mL} \mathrm{D}_{2} \mathrm{O}$.

The $\mathrm{PMeOxz}_{72}$-grad-PPhOxz $28+50 \%$ LSR nanocarriers were prepared by TFHM in $\mathrm{D}_{2} \mathrm{O}$ solutions by dissolving $1 \mathrm{mg}$ of the copolymer in $1 \mathrm{~mL} \mathrm{D}_{2} \mathrm{O}$. The partitioning of the drug in $\mathrm{PMeOxz}_{72}$-grad-PPhOxz 28 nanoassemblies was studied by adding an appropriate amount of LSR (to prepare $50 \mathrm{wt} . \%$ concentration of LSR) into the final copolymer-drug mixture.

\subsection{Methods}

${ }^{1} \mathrm{H}-\mathrm{NMR}$ spectroscopy studies of $\mathrm{PMeOxz}_{72}$-grad-PPhOxz 28 + 50\% LSR nanocarriers were conducted on an Agilent $(600 \mathrm{mHz})$ NMR spectrometer operated by Vjnmr software with a $5 \mathrm{~mm} \mathrm{HCN}$ cold probe. Tetramethylsilane (TMS) was used as the internal standard and $\mathrm{D}_{2} \mathrm{O}$ as the solvent. Furthermore, ${ }^{1} \mathrm{H}$ NMR spectra were recorded with 65,536 points, $90^{\circ}$ pulse, $10 \mathrm{~s}$ relaxation delay and 32 repetitions. 2D COSY, NOESY and DOSY experiments were performed on $600 \mathrm{MHz}$ NMR spectrometer. Specifically, NOESY spectra were recorded at different mixing times with $4096 \times 200$ points, $1 \mathrm{~s}$ relaxation delay and 32 repetitions per spectrum. The DgcsteSL_cc sequence was used to record DOSY spectra with 65,536 points, $1 \mathrm{~s}$ relaxation delay and 16 repetitions. 24 gradient strengths between zero and 60 gauss $/ \mathrm{cm}$ were used. Chemical shifts are referenced with respect to the lock frequency and reported relative to TMS. Temperature studies of $\mathrm{PMeOxz}_{72}$-grad- 
PPhOxz 28 GC were performed on a Varian $300(300 \mathrm{mHz})$ spectrometer. The composition of $\mathrm{PMeOxz}_{72}$-grad-PPhOxz 28 GC was reported in a previous work [30].

DLS measurements were carried out using an ALV/CGS-3 Compact Goniometer System (ALV GmbH, Siemensstraße 4, 63225 Langen (Hessen), Germany) equipped with an ALV-5000/EPP multi-tau digital correlator of 288 channels and an ALV/LSE-5003 light scattering electronic unit for stepper motor drive and limit switch control. Moreover, a JDS Uniphase 22-mW He-Ne laser (632.8 nm) was used as the light source. All figures exhibited in the manuscript are from averaged measurements at 90 degrees, (five measurements per concentration/angle). The cumulants method and CONTIN software were used in order to perform the analysis of autocorrelation functions. All solutions were filtered through $0.45 \mu \mathrm{m}$ hydrophilic PTFE filters (Millex-LCR from Millipore, Billerica, MA, USA) before measurements.

ELS measurements were pursued on a ZetaSizer Nanoseries Nano-ZS (Malvern Instruments Ltd., Malvern, UK) equipped with a 4-mW solid-state laser at a wavelength of $633 \mathrm{~nm}$ and a fixed backscattering angle of $173^{\circ}$. The obtained zeta-potential $\left(\zeta_{\text {pot }}\right)$ values are the average values of 50 runs, utilizing the Henry approximation of Smoluchowski equation after equilibration at $25^{\circ} \mathrm{C}$.

UV-Vis absorption spectra of LSR and $\mathrm{PMeOxz}_{72}$-grad-PPhOxz 28 nanocarriers were recorded between 200 and $600 \mathrm{~nm}$ wavelength using a Perkin Elmer (Lambda 19) UV-VisNIR spectrophotometer (Waltham, MA, USA). The LSR-loaded PMeOxz72-grad-PPhOxz 28 nanocarriers were diluted to get an absorbance value of less than 1 . It is notable that the absorption at $260 \mathrm{~nm}$ is related to the presence of LSR and not of the copolymer based on measurements on the pure components.

Mid-infrared (IR) spectra of LSR, PMeOxz 72 -grad-PPhOxz 28 nanoassemblies and LSRloaded $\mathrm{PMeOxz}_{72}$-grad-PPhOxz 28 nanocarriers were recorded in the region $550-4000 \mathrm{~cm}^{-1}$ on a FTIR spectrometer (Equinox 55 from Bruker Optics) equipped with a single reflection diamond ATR accessory (DuraSamp1IR II by SensIR Technologies).

ATR-FTIR spectral peaks of PMeOxz 72 -grad-PPhOxz 28 nanoassemblies: $\mathrm{v}\left(\mathrm{cm}^{-1}\right)=$ 2820-3050 (-CH-), 1639 (-C=O-), 1517-1335 (-N-CH ${ }_{2}-$ ), 1335-900 (-C-N-), 815-560 (aromatic C-H).

\section{Results and Discussion}

\subsection{Synthesis and Molecular Characterization of PMeOxz $z_{72}-$ Grad-PPhOxz $z_{28}$ GC}

The synthetic procedure and molecular characterization of the amphiphilic PMeOxz72grad-PPhOxz 28 GC is outlined in the study by Milonaki et al. [30]. The molecular characteristics are exhibited in Table 1 and the chemical structures of the copolymer and LSR are presented in Scheme 1.

Table 1. Molecular characteristics of the PMeOxz72-grad-PPhOxz 28 GC.

\begin{tabular}{|c|c|c|c|c|}
\hline Sample & $\begin{array}{c}\operatorname{Mn}^{a} \\
(\mathrm{~g} / \mathrm{mol})\end{array}$ & $\begin{array}{c}\mathrm{Mn}^{\mathrm{b}} \\
(\mathrm{g} / \mathrm{mol})\end{array}$ & $\mathbf{M}_{\mathrm{w}} / \mathbf{M}_{\mathbf{n}}{ }^{\mathbf{b}}$ & $\% \mathrm{PPhOxz}^{\mathrm{a}}$ \\
\hline $\mathrm{PMeOxz}_{72}$-grad-PPhOxz 28 & 4900 & 5200 & 1.14 & 28 \\
\hline
\end{tabular}

a Determined by ${ }^{1} \mathrm{H}-\mathrm{NMR}$ in $\mathrm{CDCl}_{3} \cdot{ }^{\mathrm{b}}$ Determined by SEC in $\mathrm{CHCl}_{3}$ using polystyrene standards. 


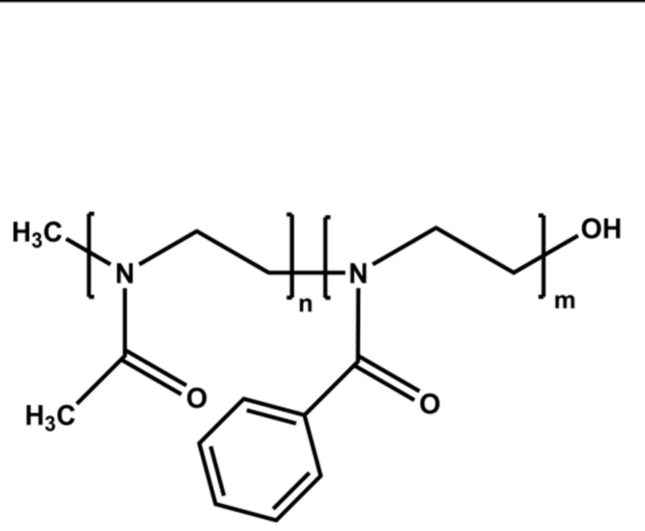

(a) PMeOxz-grad-PPhOxz

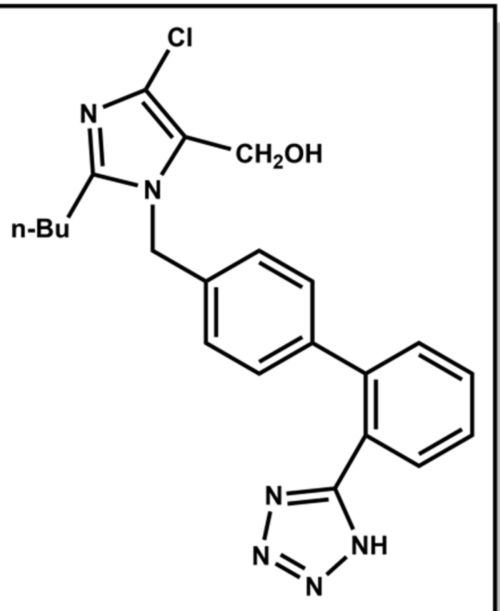

(b) Losartan

Scheme 1. Chemical structures of (a) PMeOxz72-grad-PPhOxz 28 GC synthesized by cationic polymerization and (b) LSR.

\subsection{Physicochemical Characterization of $P M e O x z_{72}-G r a d-P P h O x z_{28} G C$}

A detailed overview of the size, shape, surface charge and chemical structure of the $\mathrm{PMeOxz}_{72}$-grad-PPhOxz 28 GC is provided using DLS, ELS and ATR-FTIR physicochemical techniques. Based on the literature, the PMeOxz72-grad-PPhOxz 28 GC solution in water exhibited three populations that differ in size, i.e., the unimers, the micelles and the aggregates [30]. The DLS data in the size distribution plot of Figure 1a, are in good agreement with other scientific reports [30,42]. Particularly, Figure 1a reveals the existence of three different populations in size and intensity, presumably corresponding to free GC chains $\left(R_{h}=3 \mathrm{~nm}\right)$, micelles $\left(R_{h}=13 \mathrm{~nm}\right)$ and aggregates of larger size $\left(R_{h}=96 \mathrm{~nm}\right)$. The intensity weighted distribution of the latter was found to be the most prominent. The presence of unimers is presumably linked to the low molecular weight of the copolymer and its gradient structure. Previous studies have demonstrated that copolymer chains involving some mixing of the monomers tend to exhibit relatively low aggregation tendencies $[43,44,47-50]$. The formation of large aggregates is possibly related to the gradient structure of the copolymer chains that maximize the exposure of hydrophobic PPhOxz segments to water, thus enhancing the hydrophobic interactions of micelles. Likewise, the quite polydisperse $(\mathrm{PDI}=0.48)$ population of large aggregates may be attributed to the gradient structure of the copolymer which imposes some spatial constrains in the arrangement of macromolecules leading to better defined nanostructures. The hydrodynamic radius $\left(R_{h}\right)$ and size polydispersity index (PDI) values of the PMeOxz 72 -grad-PPhOxz 28 GC formed in aqueous media at $\mathrm{C}=10^{-3} \mathrm{~g} / \mathrm{mL}, \mathrm{pH}=7$ and $25^{\circ} \mathrm{C}$ are summarized in Table 2 . The $\zeta_{\text {pot }}$ potential values of $\mathrm{PMeOxz}_{72}$-grad-PPhOxz 28 GC in aqueous solutions were around zero (Table 2) according to ELS measurements, because of the absence of net charges on nanostructures' surface.

The ATR-FTIR analysis of $\mathrm{PMeOxz}_{72}$-grad-PPhOxz 28 nanoassemblies certified their chemical structure in aqueous media. In particular, the ATR-FTIR spectrum of the nanoassemblies presented in Figure 1b), reveals several characteristic absorption bands of the expected molecular structure of the material. The peaks at $2820-3050 \mathrm{~cm}^{-1}$ and $1639 \mathrm{~cm}^{-1}$ are attributed to the stretching vibrations of aliphatic $\mathrm{C}-\mathrm{H}$ region and carbonyl $\mathrm{C}=\mathrm{O}$ group in the amide functionality of both monomeric units. The peaks centered at $1517-1335 \mathrm{~cm}^{-1}$, correspond to the asymmetric stretching vibrations of the $\mathrm{N}-\mathrm{CH}_{2}$ groups on the polymer chain. In addition, the peaks located at $1335-900 \mathrm{~cm}^{-1}$ are referred to the C-N stretching bond vibrations in the amide moieties and finally the peaks at $815-560 \mathrm{~cm}^{-1}$ to the aromatic C-H out-of-plane bending vibrations of the phenyl groups of the monomeric units. 

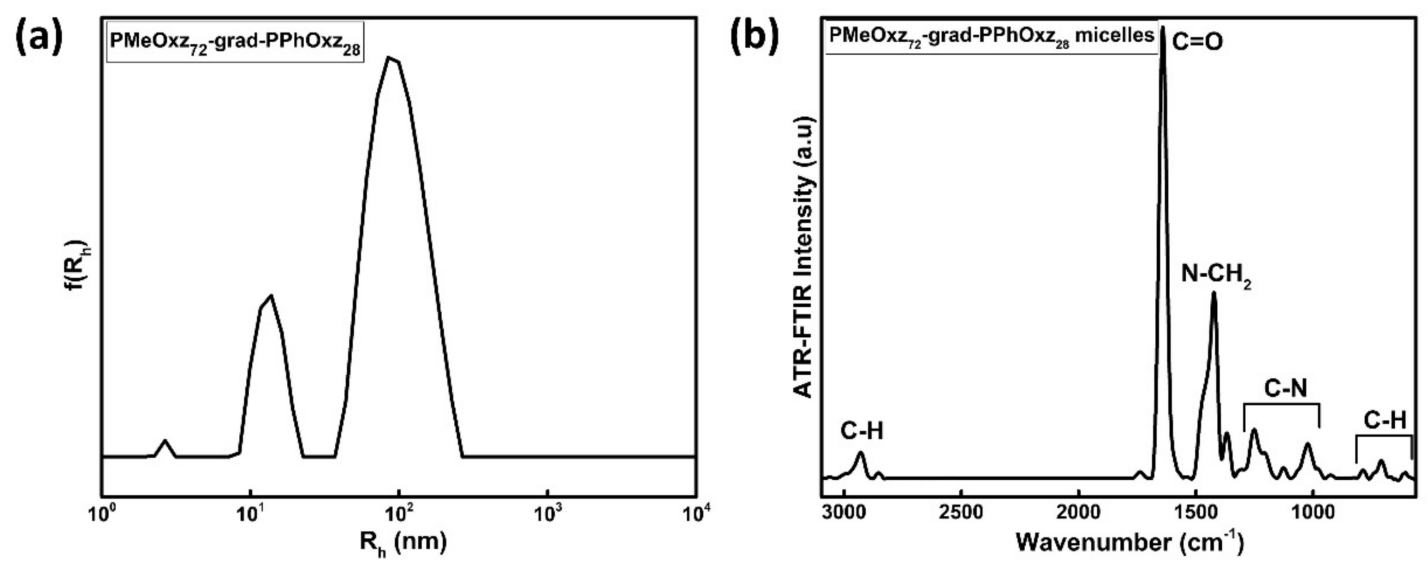

Figure 1. (a) Size distribution from Contin of $\mathrm{PMeOxz}_{72}$-grad-PPhOxz 28 GC at $90^{\circ}$ in aqueous solutions using the TFHM, (b) ATR-FTIR spectra of PMeOxz 72 -grad-PPhOxz 28 nanoassemblies in aqueous solutions using the TFHM.

Table 2. DLS and ELS results for the PMeOxz72-grad-PPhOxz 28 copolymer and LSR-loaded $\mathrm{PMeOxz}_{72}$-grad-PPhOxz 28 nanocarriers in aqueous solutions.

\begin{tabular}{|c|c|c|c|c|}
\hline Sample & $R_{h}{ }^{a}(n m)$ & PDI $^{a}$ & Intensity ${ }^{a}$ (a.u) & $\zeta_{\text {pot }} b(\mathrm{mV})$ \\
\hline \multirow{3}{*}{$\mathrm{PMeOxz}_{72}$-grad-PPhOxz 28} & 4 & \multirow{3}{*}{0.48} & \multirow{3}{*}{721} & \multirow{3}{*}{+4} \\
\hline & 13 & & & \\
\hline & 96 & & & \\
\hline \multirow{2}{*}{$\begin{array}{l}\mathrm{PMeOxz}_{72} \text {-grad-PPhOxz } 28 \\
\text { LSR }\end{array}$} & 12 & \multirow{2}{*}{0.29} & \multirow{2}{*}{979} & \multirow{2}{*}{+2} \\
\hline & 117 & & & \\
\hline \multirow{2}{*}{$\begin{array}{c}\mathrm{PMeOxz}_{72} \text {-grad-PPhOxz } 28 \\
\text { LSR }\end{array}$} & 10 & \multirow{2}{*}{0.43} & \multirow{2}{*}{2,444} & \multirow{2}{*}{+1} \\
\hline & 59 & & & \\
\hline
\end{tabular}

a Determined by DLS at measuring angle $90^{\circ} ;{ }^{\mathrm{b}}$ Determined by ELS at measuring angle $90^{\circ}$.

\subsection{Physicochemical Characterization of the LSR-Loaded PMeOxz $z_{72}$-Grad-PPhOxz 28 Nanocarriers}

DLS, ELS, ATR-FTIR, and UV-Vis studies were performed to determine the physicochemical characteristics of LSR-loaded $\mathrm{PMeOxz}_{72}$-grad-PPhOxz 28 nanocarriers and to examine the drug encapsulation into the structure of the $\mathrm{PMeOxz}_{72}$-grad-PPhOxz 28 nanoassemblies. The chemical structure, amphiphilic character and biocompatibility of $\mathrm{PMeOxz}_{72}-$ grad-PPhOxz ${ }_{28}$ GC contribute to the efficient encapsulation of antihypertensive LSR, which also consists of aromatic rings and amide groups, similarly to the GC. The measurements were performed at $10^{-3} \mathrm{~g} / \mathrm{mL}$ copolymer concentrations, $\mathrm{pH}=7$, and $25^{\circ} \mathrm{C}$. A comparison of size distributions of bare $\mathrm{PMeOxz}_{72}$-grad-PPhOxz 28 nanoassemblies and LSR-loaded $\mathrm{PMeOxz}_{72}$-grad-PPhOxz 28 nanocarriers prepared by TFHM is provided in Figure 2a. DLS data of PMeOxz72-grad-PPhOxz 28 + 20\% LSR and PMeOxz 72 -grad-PPhOxz 28 +50\% LSR nanocarriers reveal the formation of micelles and aggregates. The presence of hydrophobic LSR possibly alters the aggregation behavior of the GC/drug mixed systems, resulting in the formation of aggregates that may be considered as hyper micellar nanostructures. In particular, the size distribution plot of $\mathrm{PMeOxz}_{72}$-grad-PPhOxz $28+20 \%$ LSR nanocarriers in Figure 2a (red line) exhibits two populations at $R_{h}=12 \mathrm{~nm}$ and $R_{h}=117 \mathrm{~nm}$, suggesting the formation both of micelles and aggregates. Significantly, the peak assigned to GC/drug micelles is considerably more intense and narrower than the bare GC micelles (Figure 1a), implying the formation of more well-defined drug/polymer nanostructures after $20 \mathrm{wt} . \%$ LSR encapsulation into $\mathrm{PMeOxz}_{72}$-grad-PPhOxz 28 nanoassemblies. The second population assigned to GC/drug aggregates is shifted to slightly higher $R_{h}$ values $\left(R_{h}=117 \mathrm{~nm}\right)$ and appear quite less pronounced compared to bare $G C\left(R_{h}=96 \mathrm{~nm}\right)$ aggregates, due to the hydrophobic drug molecules that increase the hydrophobic character of the mixed GC nanostructures. 

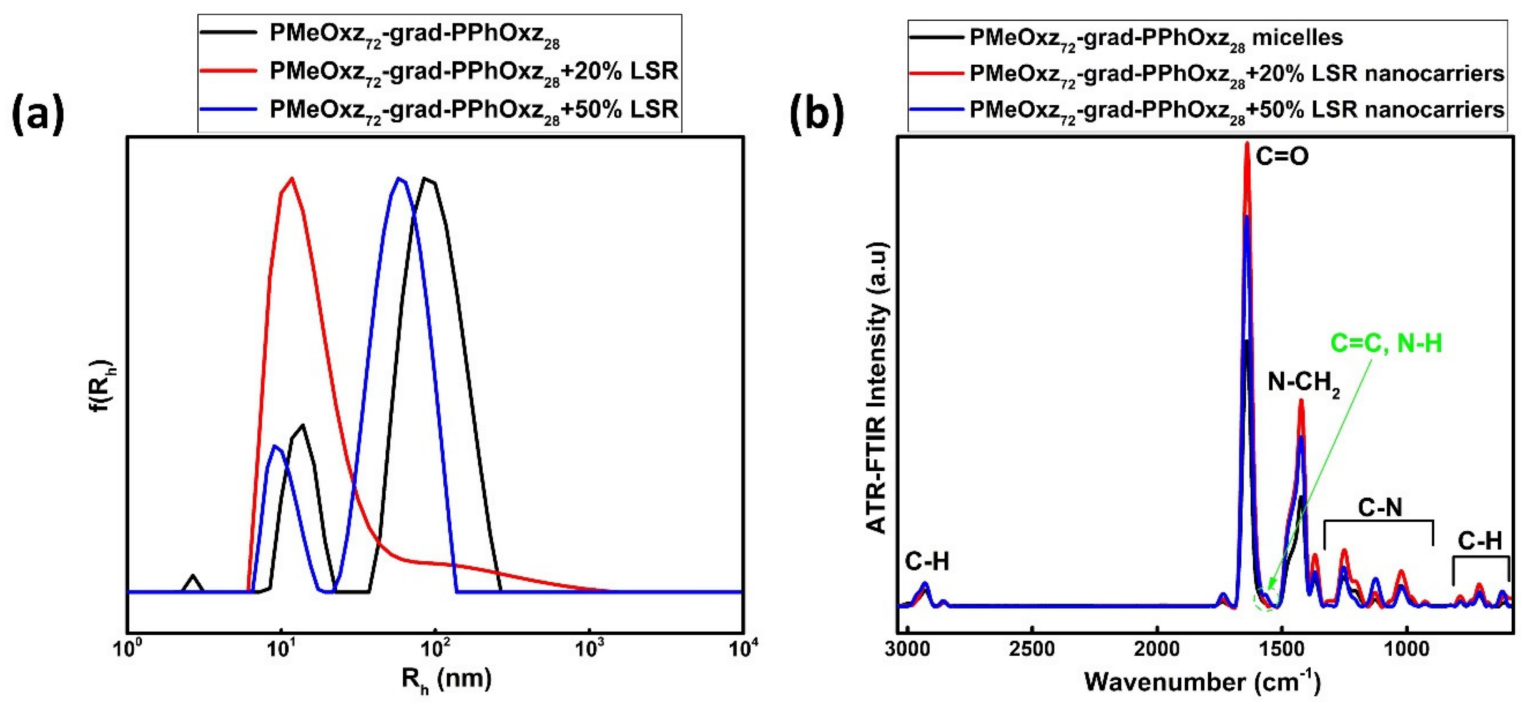

Figure 2. Comparative (a) size distributions from Contin of PMeOxz72-grad-PPhOxz 28 nanoassemblies and LSR-loaded PMeOxz72-grad-PPhOxz 28 nanocarriers at $90^{\circ}$ in aqueous solutions, (b) ATR-FTIR spectra of PMeOxz 72 -grad-PPhOxz 28 nanoassemblies and LSR-loaded PMeOxz72-grad-PPhOxz 28 nanocarriers in aqueous solutions prepared by the TFHM.

Similarly, the size distribution plot of $\mathrm{PMeOxz}_{72}$-grad-PPhOxz $28+50 \%$ LSR nanocarriers depicted in Figure 2a (blue line) denotes the formation of micelles $\left(R_{h}=10 \mathrm{~nm}\right)$ and aggregates $\left(R_{h}=60 \mathrm{~nm}\right)$. As LSR concentration increases to $50 \%$, the aggregation behavior of the $\mathrm{PMeOxz}_{72}$-grad-PPhOxz $28+50 \%$ LSR nanocarriers changes dramatically, resulting in the formation of smaller aggregates compared to PMeOxz 72 -grad-PPhOxz $28+20 \%$ LSR nanocarriers and bare GC. Apparently, the lipophilic nature of LSR alters the structural characteristics of the GC/drug mixed assemblies by modulating the organization of the polymeric components [51-54].

In addition, DLS data evidenced a significant increase in mass (based on determined scattered intensity from the solutions studied) of the LSR-loaded PMeOxz72-grad-PPhOxz 28 nanocarriers, implying a successful encapsulation of hydrophobic LSR into the $\mathrm{PMeOxz}_{72}-$ grad-PPhOxz 28 nanoassemblies, and manifested some detectable changes in the structural characteristics of the GC/drug nanostructures in comparison to the GC alone. Nevertheless, the GC/drug mixed aggregates still have sizes in the nanometer scale, which make them suitable candidates for drug delivery applications.

After loading negatively charged LSR to the GC/drug mixed assemblies, the $\zeta_{\text {pot }}$ values of $\mathrm{PMeOxz}_{72}$-grad-PPhOxz 28 + 20\% LSR nanocarriers and $\mathrm{PMeOxz}_{72}$-grad-PPhOxz 28 + $50 \%$ LSR nanocarriers slightly decreased. The values of $\mathrm{R}_{\mathrm{h}}$, PDI, scattered light intensity and $\zeta_{\text {pot }}$ of the LSR-loaded PMeOxz 72 -grad-PPhOxz 28 nanocarriers formed by TFHM in aqueous media, are summarized in Table 2.

The LSR encapsulation into the PMeOxz72-grad-PPhOxz 28 nanoassemblies was also examined by ATR-FTIR and UV-Vis measurements. A comparison of ATR-FTIR of neat $\mathrm{PMeOxz}_{72}$-grad-PPhOxz 28 nanoassemblies and LSR-loaded $\mathrm{PMeOxz}_{72}$-grad-PPhOxz 28 nanocarriers prepared by TFHM is provided in Figure 2b. A significant increase in ATRFTIR intensity peaks is noticed in the case of $\mathrm{PMeOxz}_{72}$-grad-PPhOxz $28+20 \% \mathrm{LSR}$ (red line) and $\mathrm{PMeOxz}_{72}$-grad-PPhOxz $28+50 \%$ LSR nanocarriers (blue line) compared to bare nanoassemblies, denoting significant structural changes in the GC/drug system. Specifically, the notable increase in ATR-FTIR intensity peaks in the $1335-900 \mathrm{~cm}^{-1}$ and $815-560 \mathrm{~cm}^{-1}$ regions, is probably related to C-N stretching vibrations and C-H of the LSR aromatic ring. Likewise, new characteristic peaks of LSR (green annotations) are clearly observed in the ATR-FTIR spectra of the nanocarriers. In Figure $2 b$, the appearance of a new characteristic absorption peak at about $1566 \mathrm{~cm}^{-1}$ is correlated to $C=C$ stretching modes which are attributed to the phenyl rings and also to secondary amine $\mathrm{N}-\mathrm{H}$ bending 
vibrations of LSR. All data confirm the existence of LSR in the mixed GC/drug aqueous solutions.

Only a few conclusions can be drawn regarding the UV-Vis spectra of Figure 3, due to the chemical similarity between $\mathrm{PMeOxz}_{72}$-grad-PPhOxz 28 and LSR. Figure 3 presents the UV-Vis spectra of $\mathrm{PMeOxz}_{72}$-grad-PPhOxz $28+20 \%$ LSR (black line) and PMeOxz 72 -gradPPhOxz $28+50 \%$ LSR nanocarriers (red line) prepared by TFHM in aqueous solutions along with the corresponding spectra of LSR in ACTN. In line with literature data, the biphenyl ring of LSR and the phenyl group of PPhOxz exhibit maximum absorbances at about $260 \mathrm{~nm}$ wavelength [55-59]. However, the increase in the UV absorption of PMeOxz ${ }_{72}-$ grad-PPhOxz $28+50 \%$ LSR nanocarriers at $260 \mathrm{~nm}$ is related to the presence of LSR and not of the GC.

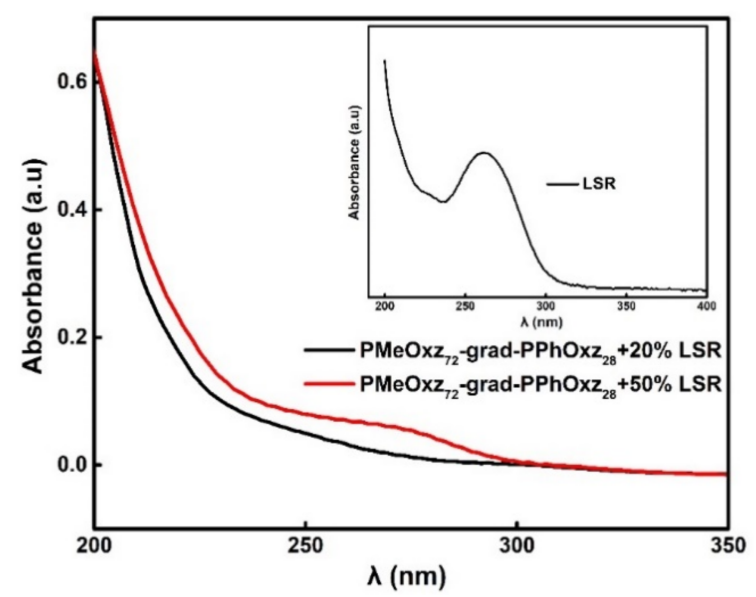

Figure 3. Comparison of UV-Vis spectra for LSR-loaded PMeOxz 72 -grad-PPhOxz 28 nanocarriers.

\subsection{FBS Interactions with PMeOxz $z_{72}$-Grad-PPhOxz $z_{28}$ GC and LSR-Loaded} PMeOxz 72 -Grad-PPhO $x z_{28}$ Nanocarriers

The stability of AmGCs nanoassemblies in serum media is vital to their use in drug delivery, where dilution after intravenous injection usually induces micellar disassembly and drug unloading $[60,61]$. In the present context, the FBS was chosen as the serum media and the physicochemical characterization of the GC and GC/drug mixed assemblies was repeated, to test if the recurring chemical/physical interactions of the nanostructures with the FBS components induce any changes to their identity.

FBS is commonly known to simulate the physicochemical conditions of blood, containing diverse proteins, mostly bovine serum albumin (BSA) (approximately $2.5 \mathrm{mg} / \mathrm{mL}$ ). Particularly, DLS stability studies of $\mathrm{PMeOxz}_{72}$-grad-PPhOxz 28 nanoassemblies and LSRloaded $\mathrm{PMeOxz}_{72}$-grad-PPhOxz 28 nanocarriers in FBS solutions were performed to detect possible alterations of their physicochemical characteristics in terms of size, PDI and scattered light intensity. The physicochemical characteristics $\left(R_{h}, P D I\right.$, Intensity) of bare FBS, PMeOxz 72 -grad-PPhOxz 28 GC and protein-GC mixtures are summarized in Table 3, while the properties of LSR-loaded $\mathrm{PMeOxz}_{72}$-grad-PPhOxz 28 nanocarriers and proteinGC/drug mixtures are gathered in Table 4 . The protocol 1 (mixing of $50 \mu \mathrm{L}$ sample with (a) $3 \mathrm{~mL}$ FBS:PBS $(1 / 9 \mathrm{v} / \mathrm{v})$ and $(\mathrm{b}) 3 \mathrm{~mL}$ FBS:PBS $(1 / 1 \mathrm{v} / \mathrm{v}))$ for the preparation of protein-GC and protein-GC/drug mixtures is denoted in Figure $4 b, c$ and Figure $5 b, c$, and the protocol 2 (mixing of $100 \mu \mathrm{L}$ sample with (a) $3 \mathrm{~mL}$ FBS:PBS (1/9 v/v) and (b) $3 \mathrm{~mL}$ FBS:PBS (1/1 v/v)) in Figure 4d,e and Figure 5d,e. 
Table 3. Physicochemical characteristics of bare FBS, PMeOxz 72 -grad-PPhOxz 28 GC and protein-GC mixtures obtained from DLS measurements based on the protocol used for their preparation. Data for $R_{h}$ denote multiple peaks.

\begin{tabular}{|c|c|c|c|c|}
\hline Sample & Protocol & $\mathbf{R}_{\mathrm{h}}(\mathrm{nm})$ & PDI & Intensity (a.u) \\
\hline FBS & - & $\begin{array}{c}4 \\
30\end{array}$ & 0.51 & 5760 \\
\hline $\mathrm{PMeOxz}_{72}$-grad-PPhOxz 28 & PBS & $\begin{array}{c}9 \\
150\end{array}$ & 0.40 & 440 \\
\hline $\begin{array}{c}\text { PMeOxz }_{72} \text {-grad-PPhOxz } 28 \\
(1: 9 \mathrm{v} / \mathrm{v})\end{array}$ & 1 & $\begin{array}{c}7 \\
74\end{array}$ & 0.51 & 861 \\
\hline $\begin{array}{c}\text { PMeOxz }_{72} \text {-grad-PPhOxz } \\
(1: 1 \mathrm{v} / \mathrm{v})\end{array}$ & 1 & $\begin{array}{c}2 \\
8.5 \\
84\end{array}$ & 0.50 & 3012 \\
\hline $\begin{array}{c}\text { PMeOxz }_{72} \text {-grad-PPhOxz } \\
(1: 9 \mathrm{v} / \mathrm{v})\end{array}$ & 2 & $\begin{array}{c}2 \\
8 \\
65\end{array}$ & 0.52 & 751 \\
\hline $\begin{array}{c}\text { PMeOxz }_{72} \text {-grad-PPhOxz } \\
(1: 1 \mathrm{v} / \mathrm{v})\end{array}$ & 2 & $\begin{array}{c}3 \\
10 \\
60\end{array}$ & 0.52 & 2970 \\
\hline
\end{tabular}

Table 4. Physicochemical characteristics of LSR-loaded PMeOxz 72 -grad-PPhOxz 28 nanocarriers and protein-GC/drug mixtures obtained from DLS measurements based on the protocol used for their preparation. Data for $\mathrm{R}_{\mathrm{h}}$ denote multiple peaks.

\begin{tabular}{|c|c|c|c|c|}
\hline Sample & Protocol & $\mathrm{R}_{\mathrm{h}}(\mathrm{nm})$ & PDI & Intensity (a.u) \\
\hline $\mathrm{PMeOxz}_{72}$-grad-PPhOxz $28+20 \%$ LSR & PRC & 29 & 0.43 & 271 \\
\hline $\mathrm{PMeOxz}_{72}$-grad-PPhOxz 28 + 50\% LSR & PBS & 12 & 0.3 & 141 \\
\hline $\begin{array}{c}\text { PMeOxz72-grad-PPhOxz } 28+20 \% \mathrm{LSR}+ \\
\text { FBS:PBS }(1: 9 \mathrm{v} / \mathrm{v})\end{array}$ & 1 & $\begin{array}{c}3 \\
9 \\
78\end{array}$ & 0.46 & 1032 \\
\hline $\begin{array}{c}\text { PMeOxz } 72 \text {-grad-PPhOxz } 28+20 \% \text { LSR + } \\
\text { FBS:PBS }(1: 1 \mathrm{v} / \mathrm{v})\end{array}$ & 1 & $\begin{array}{c}3 \\
11 \\
82\end{array}$ & 0.47 & 1061 \\
\hline $\begin{array}{c}\text { PMeOxz } 72 \text {-grad-PPhOxz } 28+20 \% \mathrm{LSR}+ \\
\text { FBS:PBS }(1: 9 \mathrm{v} / \mathrm{v})\end{array}$ & 2 & $\begin{array}{c}2 \\
8 \\
77\end{array}$ & 0.49 & 1019 \\
\hline $\begin{array}{c}\mathrm{PMeOxz}_{72} \text {-grad-PPhOxz } \\
\text { FBS:PBS }(1: 1 \mathrm{v} / \mathrm{v})\end{array}$ & 2 & $\begin{array}{c}3 \\
9 \\
82\end{array}$ & 0.47 & 964 \\
\hline $\begin{array}{c}\text { PMeOxz }_{72} \text {-grad-PPhOxz } \\
\text { FBS:PBS }(1: 9 \mathrm{v} / \mathrm{v})\end{array}$ & 1 & $\begin{array}{c}3 \\
10 \\
77\end{array}$ & 0.50 & 653 \\
\hline $\begin{array}{c}\text { PMeOxz }_{72} \text {-grad-PPhOxz } \\
\text { FBS:PBS }(1: 1 \mathrm{v} / \mathrm{v})\end{array}$ & 1 & $\begin{array}{c}3 \\
11 \\
97\end{array}$ & 0.52 & 3051 \\
\hline $\begin{array}{c}\text { PMeOxz } 72 \text {-grad-PPhOxz } 28+50 \% \mathrm{LSR}+ \\
\text { FBS:PBS }(1: 9 \mathrm{v} / \mathrm{v})\end{array}$ & 2 & $\begin{array}{c}3 \\
12 \\
96\end{array}$ & 0.50 & 672 \\
\hline $\begin{array}{c}\text { PMeOxz } 72 \text {-grad-PPhOxz } 28+50 \% \text { LSR + } \\
\text { FBS:PBS }(1: 1 \mathrm{v} / \mathrm{v})\end{array}$ & 2 & $\begin{array}{c}3 \\
10 \\
86\end{array}$ & 0.51 & 2974 \\
\hline
\end{tabular}



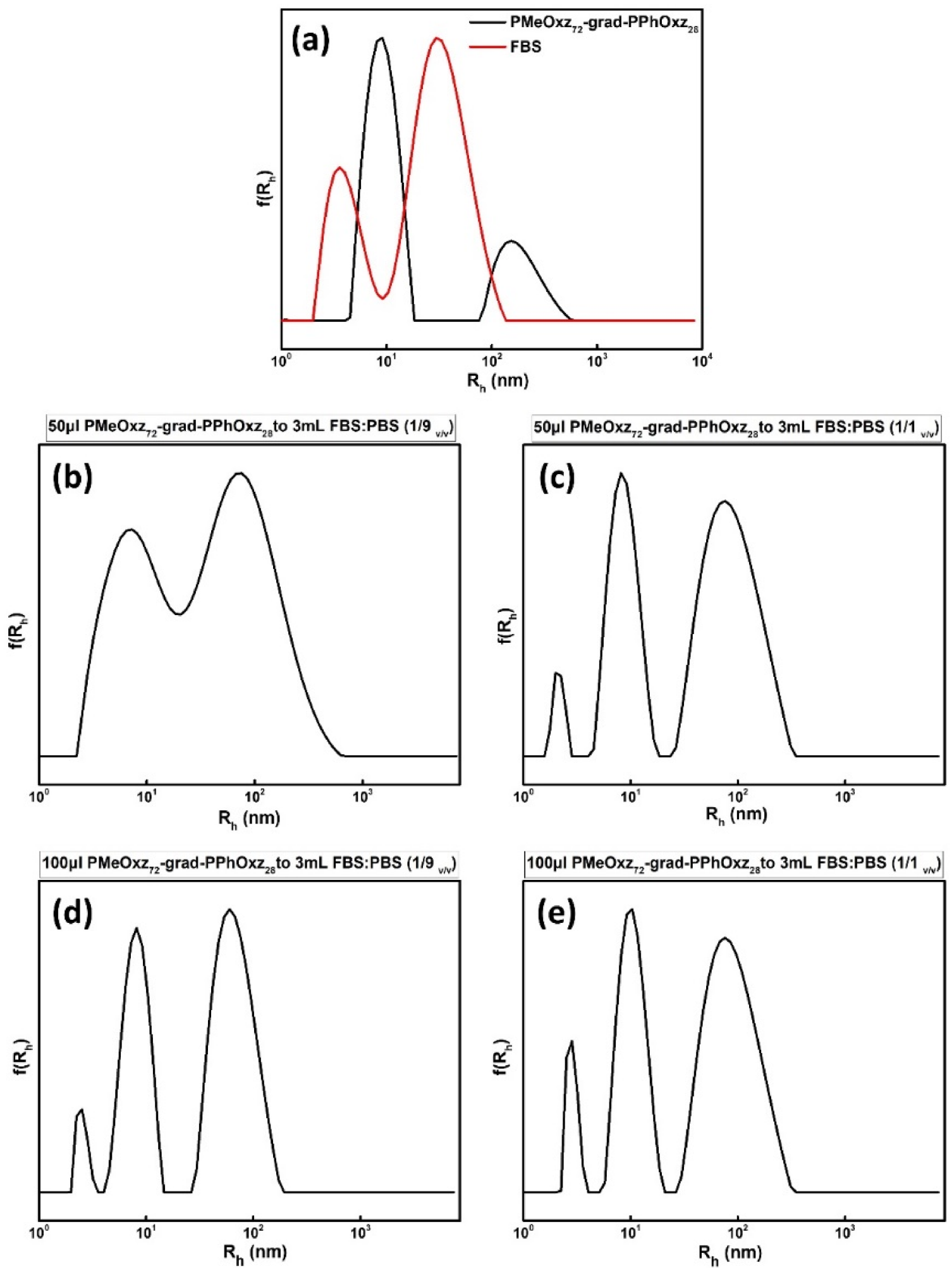

Figure 4. (a) Comparative size distributions of bare PMeOxz 72 -grad-PPhOxz 28 nanoassemblies in PBS and bare FBS and intensity size distributions of (b) PMeOxz72-grad-PPhOxz 28 + FBS:PBS (1/9 v/v) using protocol 1, (c) PMeOxz72-gradPPhOxz $28+$ FBS:PBS (1/1 v/v) using protocol 1, (d) PMeOxz 72 -grad-PPhOxz $28+$ FBS:PBS (1/9 v/v) using protocol 2 and (e) PMeOxz72-grad-PPhOxz 28 + FBS:PBS (1/1 v/v) using protocol 2. 

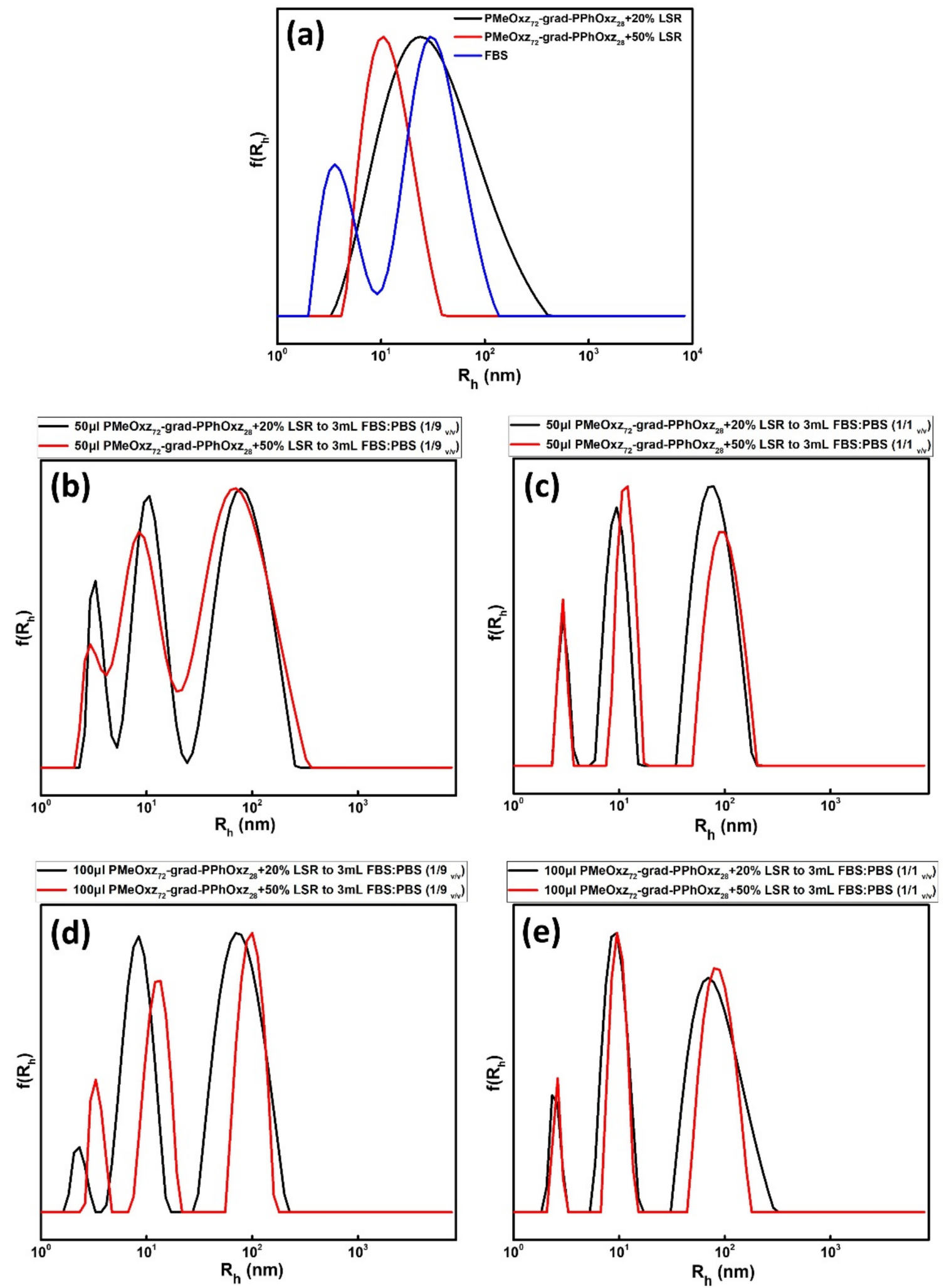

Figure 5. Comparative size distributions of (a) $\mathrm{PMeOxz} 72$-grad-PPhOxz $28+20 \% \mathrm{LSR}, \mathrm{PMeOxz}_{72}$-grad-PPhOxz $28+50 \%$ LSR nanocarriers and bear FBS, (b) PMeOxz 72 -grad-PPhOxz $28+20 \%$ LSR + FBS:PBS (1/9 v/v) and PMeOxz 72 -gradPPhOxz 28 + 50\% LSR + FBS:PBS (1/9 v/v) using protocol 1, (c) PMeOxz 72 -grad-PPhOxz $28+20 \%$ LSR + FBS:PBS (1/1 v/v) and PMeOxz 72 -grad-PPhOxz $28+50 \%$ LSR + FBS:PBS (1/1 v/v) using protocol 1, (d) PMeOxz 72 -grad-PPhOxz $28+20 \%$ LSR + FBS:PBS (1/9 v/v) and PMeOxz72-grad-PPhOxz 28 + 50\% LSR+FBS:PBS (1/9 v/v) using protocol 2, (e) PMeOxz72grad-PPhOxz 28 + 20\% LSR+FBS:PBS (1/1 v/v) and PMeOxz 72 -grad-PPhOxz 28 + 50\% LSR + FBS:PBS (1/1 v/v) using protocol 2. 
The intensity size distributions of bare $\mathrm{PMeOxz}_{72}$-grad-PPhOxz 28 nanoassemblies in PBS (black line) and bare FBS (red line) are presented in Figure 4a. Accordingly, Figure 5a presents the intensity size distributions of $\mathrm{PMeOxz}_{72}$-grad-PPhOxz $28+20 \%$ LSR (black line) and $\mathrm{PMeOxz} 72$-grad-PPhOxz 28 +50\% LSR nanocarriers (red line) in PBS. The size distribution plot of $\mathrm{PMeOxz}_{72}$-grad-PPhOxz 28 GC in PBS media (Figure 4a, black line) reveals the existence of an intense peak at $9 \mathrm{~nm}$ and of a relatively less pronounced peak at $150 \mathrm{~nm}$ which are attributed to micelles and large aggregates, respectively. In contrast to water solutions (Figure 1a), the PMeOxz72-grad-PPhOxz 28 GC forms mainly micelles in PBS, as a small percentage of the aggregates is evident in the size distribution plot of Figure 4a (black line). However, large aggregates are not observed in the comparative size distributions of $\mathrm{PMeOxz}_{72}$-grad-PPhOxz $28+20 \%$ LSR and PMeOxz 72 -grad-PPhOxz 28 $+50 \%$ LSR solutions (Figure 5a) in PBS, which appear symmetrical and monomodal, indicating the participation of all chains in the formation of micellar nanostructures. The different size distributions in water and PBS solutions may be attributed to the differing $\mathrm{pH}$ and ionic strength of PBS, which perturb the solvation state of polymeric chains since ions are present in the solution $[62,63]$.

The great heterogeneity of proteins in FBS elicited a bimodal size distribution with peaks at $4 \mathrm{~nm}$ and $30 \mathrm{~nm}$, underlying the existence of BSA at low values of $R_{h}$ and aggregates, respectively. The characteristic peak of BSA in most DLS distributions of Figures 4 and 5 is attributed to unbound proteins. After $1 \mathrm{~h}$ of PMeOxz 72 -grad-PPhOxz 28 incubation with FBS, DLS stability studies evidenced a coexistence of free proteins and PMeOxz72-grad-PPhOxz 28 nanoassemblies (Figure 4b-e). No major interactions between PMeOxz72-grad-PPhOxz 28 nanoassemblies and FBS (for both protocols) are reported, as the overall size $\left(R_{h}\right)$ of the nanoassemblies remained almost constant before and after mixing with serum proteins. Likewise, after mixing LSR-loaded PMeOxz72-grad-PPhOxz 28 nanocarriers with serum components, free proteins, micellar nanostructures (of decreased size in the case of PMeOxz72-grad-PPhOxz $28+20 \%$ LSR) and aggregates were formed (Figure 5b-e), implying weak interactions with FBS.

In terms of scattered light intensity (Tables 3 and 4), a significant decrease in FBS is noticed when comparing protein-GC and protein-GC/drug mixtures using both protocols at $3 \mathrm{~mL}$ FBS:PBS $(1 / 9 \mathrm{v} / \mathrm{v})$ ratio, implying a mass decrease of initial FBS aggregates in most cases due to the presence of the GC nanostructures.

All data manifested weak interactions between FBS, PMeOxz72-grad-PPhOxz 28 nanoassemblies and LSR-loaded PMeOxz72-grad-PPhOxz 28 nanocarriers. Collectively, DLS observations support the idea that PMeOxz hydrophilic chains shield PMeOxz72-grad$\mathrm{PPhOxz}_{28} \mathrm{GC}$ against massive protein absorption and particle agglomeration.

\subsection{Physical Stability of LSR-Loaded PMeOxz $z_{2}$-Grad-PPhOxz 28 Nanocarriers over Time}

The physical stability over time of LSR-loaded PMeOxz 72 -grad-PPhOxz 28 solutions was assessed by measuring their size and mass for a period of 23 days using DLS. The $R_{h}$ and scattered intensity measurements (from cumulant analysis) versus time of LSR-loaded PMeOxz72-grad-PPhOxz 28 nanocarriers are juxtaposed in Figure 6. The PMeOxz 72 -grad$\mathrm{PPhOxz}_{28}+20 \%$ LSR (Figure 6a) and PMeOxz 72 -grad-PPhOxz 28 +50\% LSR nanocarriers (Figure $6 b$ ) do not exhibit significant $R_{h}$ fluctuations indicating stable mixed nanostructures, while slight variations in scattered intensity are present within 23 days. Specifically, the $R_{h}$ of both nanocarriers was steeply increased on the 3rd day, translated as a significant initial growth in the size of the mixed nanostructures (by $65 \mathrm{~nm}$ and $21 \mathrm{~nm}$ for the PMeOxz 72 -grad$\mathrm{PPhOxz}_{28}+20 \%$ LSR and PMeOxz 72 -grad-PPhOxz 28 + 50\% LSR nanocarriers, respectively). Both nanocarriers retained their size after the 3rd day up until the 23rd day, implying the formation of rather stable nanostructures. Notably, the mixed GC/drug solutions exhibited great long-term stability, as no visually detectable (with naked eye) drug precipitation was observed in a period of one year. Apparently, the corona formed by the hydrophilic PMeOxz segments of the GC effectively stabilized the resulting nanostructures. 

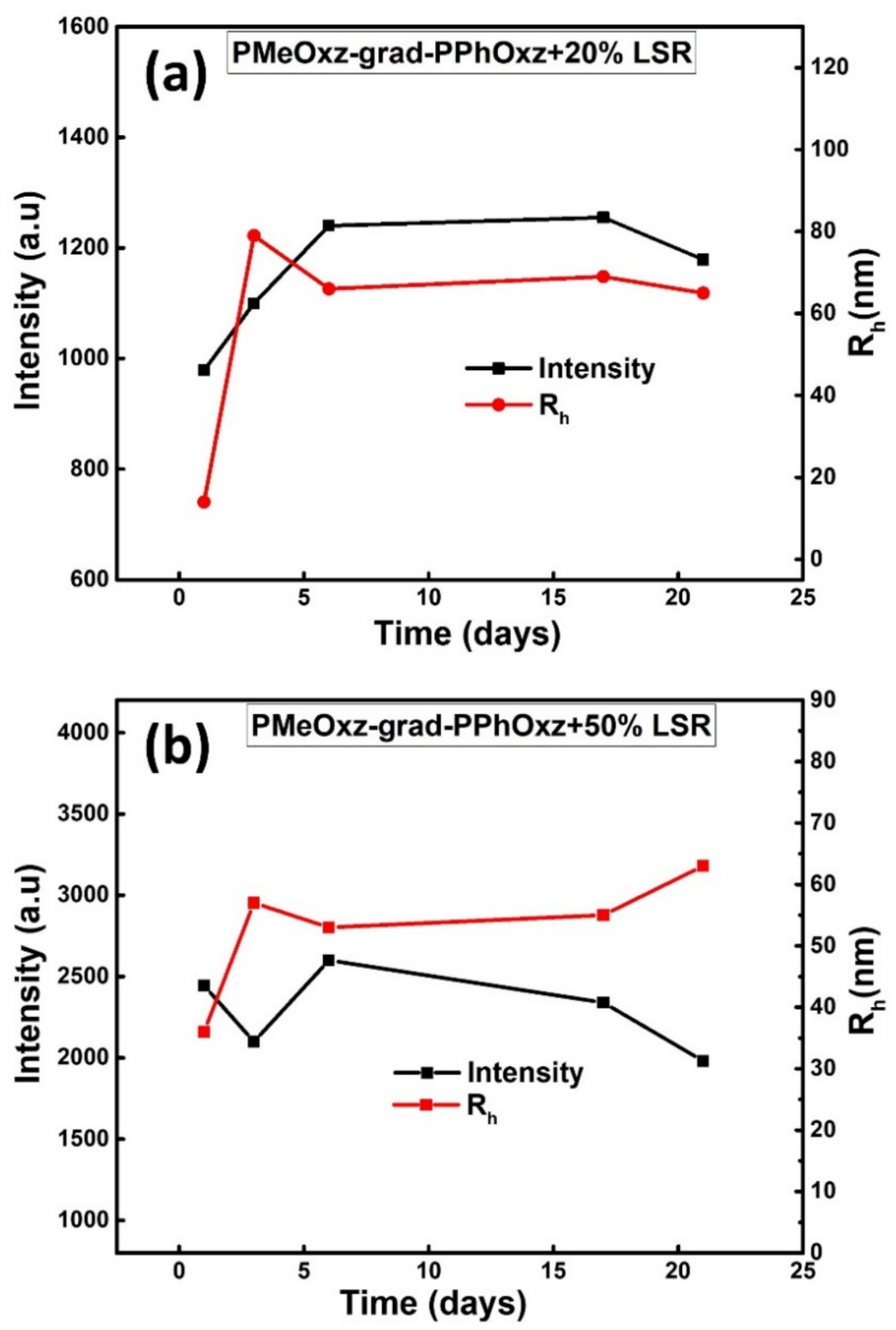

Figure 6. Scattered intensity and $R_{h}$ measurements (from cumulant analysis) versus time obtained from DLS of (a) PMeOxz 72 -grad-PPhOxz 28 + 20\% LSR and (b) PMeOxz72-grad-PPhOxz 28 +50\% LSR nanocarriers in aqueous solutions.

\subsection{NMR Studies}

A plethora of NMR techniques was utilized for the structural elucidation of GC and LSR and the verification of the successful loading of LSR into the GC/drug solutions. Structural identification of $\mathrm{PMeOxz}_{72}$-grad-PPhOxz 28 GC and LSR have been reported in previous publications $[30,58]$. The ${ }^{1} \mathrm{H}-\mathrm{NMR}$ spectrum for the $\mathrm{PMeOxz}_{72}$-grad-PPhOxz $28+$ $50 \%$ LSR nanocarriers in $\mathrm{D}_{2} \mathrm{O}$ in Figure 7, shows the distinct proton signals of LSR clearly (red letters), corroborating its successful loading in the polymeric nanocarriers. Notably, broader proton signals of the LSR phenyl group arise in positions 8-9 and 10-11, compared to those exhibited in previous studies utilizing different copolymers $[45,46]$, implying stronger hydrophobic interactions between the phenyl group of $\mathrm{PMeOxz}_{72}$-grad-PPhOxz 28 and phenyl ring of LSR. The chemical shifts of LSR proton signals in the presence of $\mathrm{PMeOxz}_{72}$-grad-PPhOxz 28 nanoassemblies, PnBA 30 -b-POEGA ${ }_{70}$ [46] and PEO-b-PCL [45] micelles are gathered in Table 5, reflecting its great molecular flexibility and the different extent of interactions with different copolymers. 


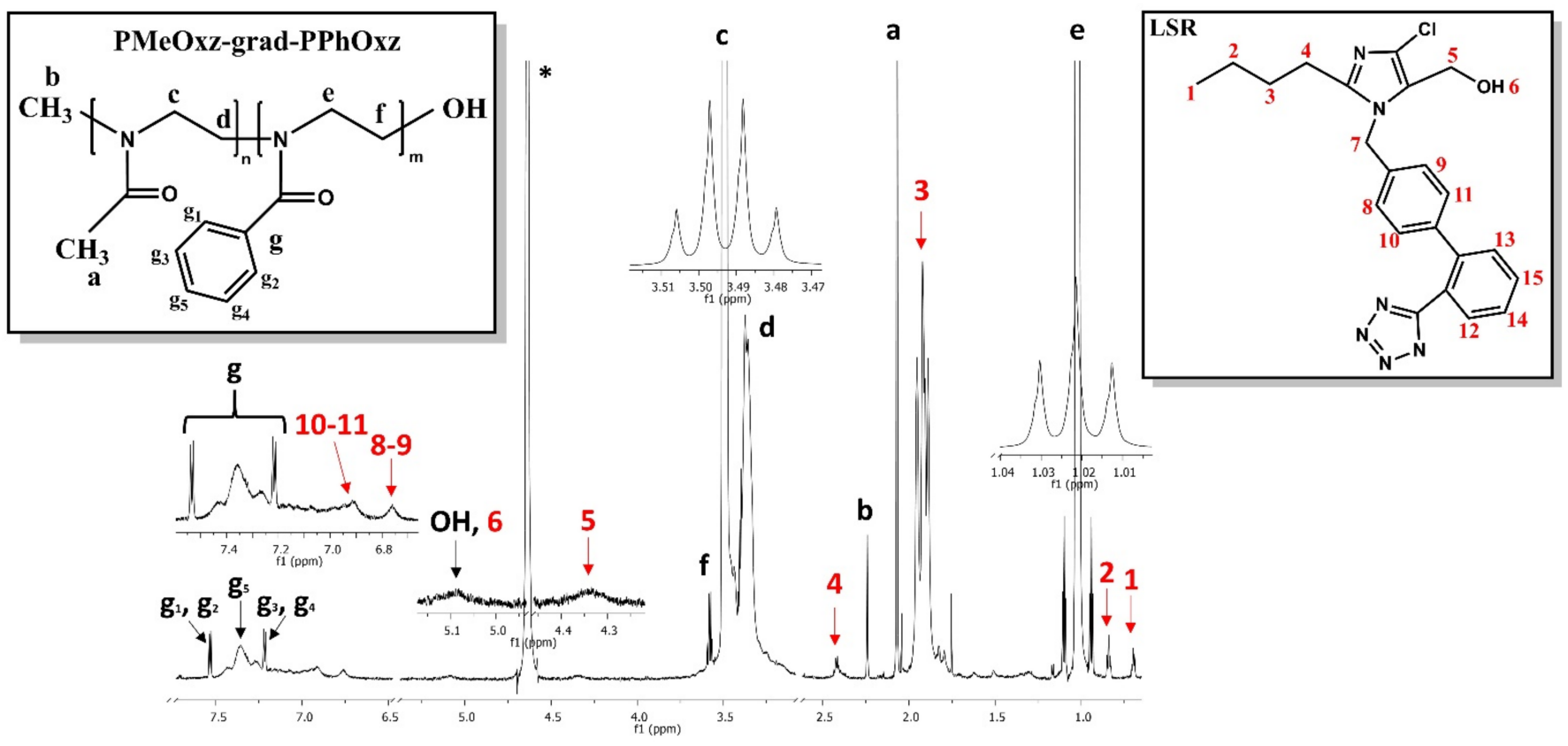

Figure 7. ${ }^{1} \mathrm{H}-\mathrm{NMR}$ spectrum for the PMeOxz72-grad-PPhOxz $28+50 \%$ LSR nanocarriers in $\mathrm{D}_{2} \mathrm{O}$ solutions, where the black letters denote the protons of the copolymer structure $(\mathrm{a}-\mathrm{g})$, the red letters point to the LSR peaks and the symbol * refers to the solvent $\mathrm{D}_{2} \mathrm{O}$.

Table 5. ${ }^{1} \mathrm{H}-\mathrm{NMR}$ chemical shifts of LSR in the presence of: (a) PMeOxz72-grad-PPhOxz 28 nanoassemblies, (b) PnBA 30 -b-POEGA 70 micelles and (c) PEO-b-PCL micelles in $\mathrm{D}_{2} \mathrm{O}$ solutions.

\begin{tabular}{|c|c|c|c|}
\hline \multirow[b]{2}{*}{$\begin{array}{l}\text { Protons of LSR } \\
\text { Structure }\end{array}$} & \multicolumn{3}{|c|}{$\begin{array}{c}{ }^{1} \mathrm{H}-\mathrm{NMR} \text { Chemical } \\
\text { Shifts (ppm) }\end{array}$} \\
\hline & $\begin{array}{l}\text { (a) Proton Signals of } \\
\text { LSR in PMeOxz } \\
\text { Grad-PPhOxz } \\
\end{array}$ & $\begin{array}{l}\text { (b) Proton Signals of } \\
\text { LSR in } \\
\text { PnBA }_{30}-\mathrm{b}-\text { POEGA }\end{array}$ & $\begin{array}{l}\text { (c) Proton Signals of } \\
\text { LSR in PEO-b-PCL }\end{array}$ \\
\hline $\mathrm{H} 1$ & 0.70 & 0.63 & 0.63 \\
\hline $\mathrm{H} 2$ & 0.90 & 1.13 & 1.22 \\
\hline $\mathrm{H} 3$ & 1.90 & 1.32 & 1.32 \\
\hline $\mathrm{H} 4$ & 2.41 & 2.43 & 2.43 \\
\hline H5 & 4.34 & 4.35 & 5.12 \\
\hline $\mathrm{H} 6(\mathrm{OH})$ & 5.08 & - & 5.4 \\
\hline $\mathrm{H} 7$ & - & 5.11 & 4.35 \\
\hline H8-H9 & 6.76 & 6.78 & 6.78 \\
\hline H10-H11 & 6.91 & 6.93 & 6.93 \\
\hline H12-H13 & $*$ & 7.45 & 7.45 \\
\hline H14-H15 & * & 7.38 & 7.19 \\
\hline Reference & \multicolumn{2}{|c|}{$\mathrm{D}_{2} \mathrm{O}: 4.69$} & \\
\hline
\end{tabular}

The internal structure of PMeOxz72-grad-PPhOxz 28 GC and LSR moieties was confirmed by 2D-COSY measurements though intramolecular interaction analysis between their segments. The 2D-COSY spectrum of the PMeOxz 72 -grad-PPhOxz $28+50 \%$ LSR nanocarriers is presented in Figure 8. The cross peaks, noticed between the protons of the GC and the protons of LSR in the 2D-COSY spectrum in Figure 8, signify the intramolecular interactions and the structural elucidation of GC and LSR. 

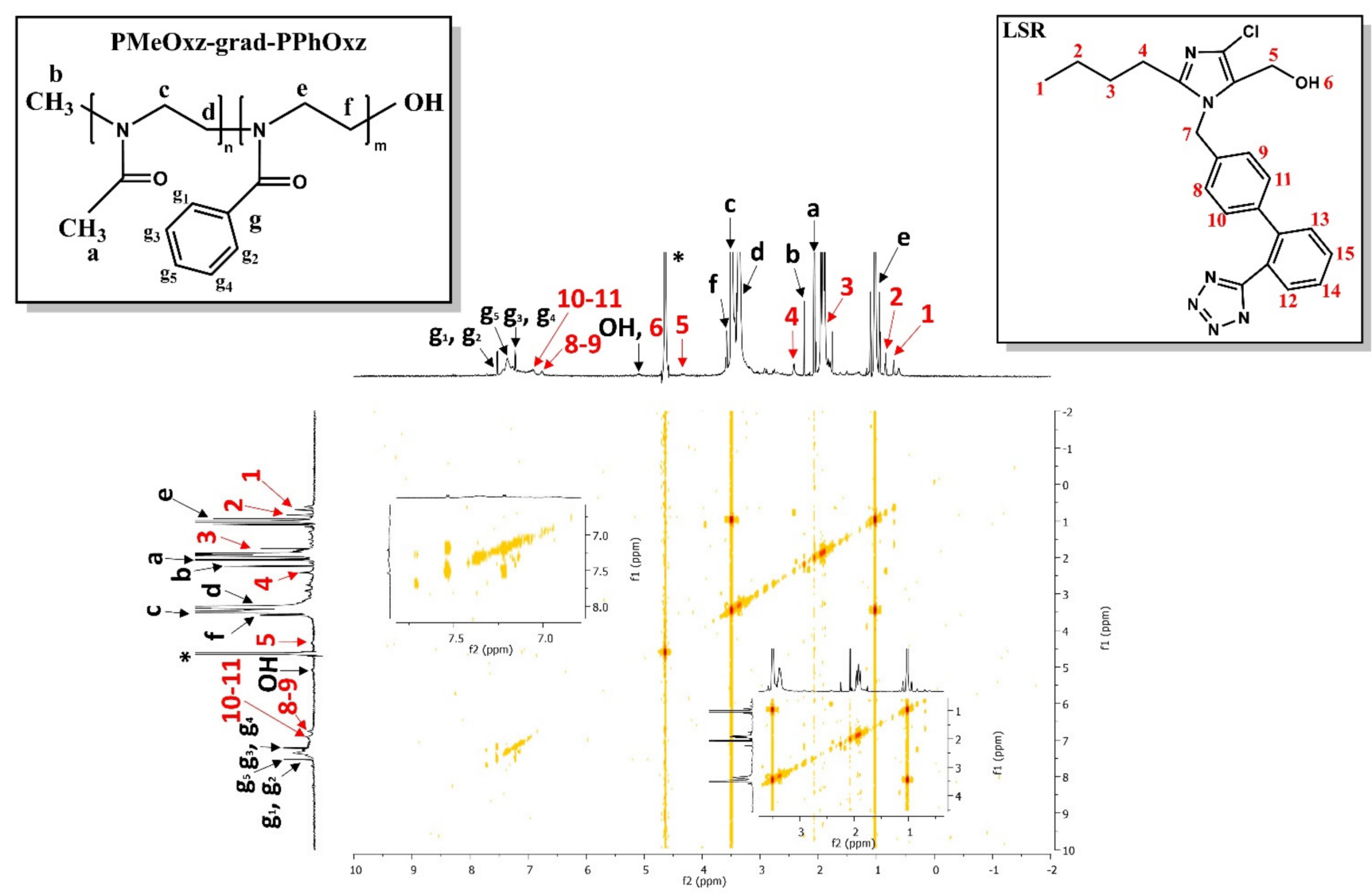

Figure 8. 2D-COSY spectrum of $\mathrm{PMeOxz}_{72}$-grad-PPhOxz $28+50 \%$ LSR nanocarriers in $\mathrm{D}_{2} \mathrm{O}$ solutions, where the black letters $(\mathrm{a}-\mathrm{g})$ denote the protons of the copolymer structure, the red letters point to the LSR peaks and the symbol * refers to the solvent $\mathrm{D}_{2} \mathrm{O}$.

2D NOESY experiments on PMeOxz 72 -grad-PPhOxz $28+50 \%$ LSR nanocarriers followed to study the spatial vicinities and intramolecular/intermolecular interactions between the GC and LSR. Little evidence for the intermolecular association was obtained from the 2D-NOESY spectrum of $\mathrm{PMeOxz}_{72}$-grad-PPhOxz 28 + 50\% LSR nanocarriers (Figure 9a) in $\mathrm{D}_{2} \mathrm{O}$ solutions, due to the intrinsic difficulty in separating signals from protons of the phenyl groups of $\mathrm{PMeOxz}_{72}$-grad-PPhOxz 28 nanoassemblies and LSR. However, Figure 9a clearly shows cross peaks between the phenyl ring of LSR with the phenyl group of PPhOxz. Also, the butyl chain of LSR displays eminent correlations between the phenyl group of PPhOxz and methylene group of PMeOxz, respectively. Particularly, the phenyl ring of LSR (10-11) is in spatial proximity with the phenyl group of PPhOxz in position $g_{5}$ while the methylene of LSR, in position 3, is in spatial proximity with the phenyl group of PPhOxz $\left(\mathrm{g}_{5}\right)$ and the methylene group of $\mathrm{PMeOxz}(\mathrm{c}, \mathrm{d})$, indicating that the hydrophobic interactions are mainly exerted by the biphenyl ring of LSR and the phenyl group of PPhOxz. Figure $9 \mathrm{~b}$ illustrates the approach of LSR towards $\mathrm{PMeOxz}_{72}$-grad-PPhOxz 28 nanoassemblies. 


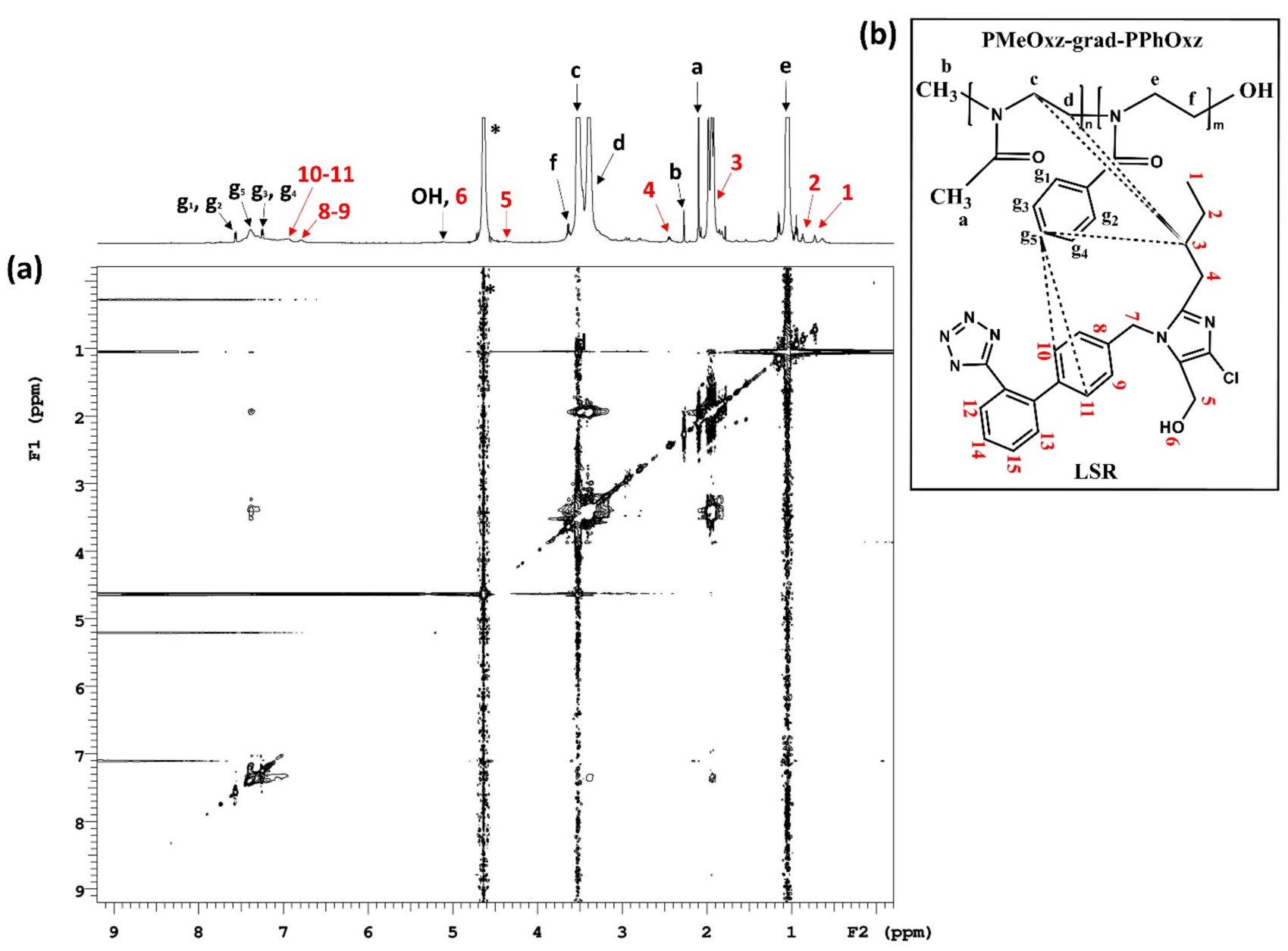

Figure 9. (a) 2D-NOESY spectrum of PMeOxz 72 -grad-PPhOxz 28 +50\% LSR nanocarriers and (b) schematic illustrating the interactions of LSR in PMeOxz72-grad-PPhOxz 28 nanoassemblies. The black letters $(\mathrm{a}-\mathrm{g})$ denote the protons of the copolymer structure, the red letters point to the LSR peaks and the symbol * refers to the solvent $\mathrm{D}_{2} \mathrm{O}$.

The PMeOxz 72 -grad-PPhOxz $28+50 \%$ LSR nanocarriers were further characterized using 2D DOSY experiments to determine the self-diffusion coefficients D, detect the presence of assemblies and probe the association of the drug with the nanoassemblies. Diffusion experiments displayed in Figure 10 confirmed the association of LSR with PMeOxz $\mathrm{P}_{72}$-grad$\mathrm{PPhOxz}_{28}$. The black arrows depicted in Figure 10 denote the protons of the copolymer structure and the red arrows point to the LSR peaks. Specifically, a triple series of traces with medium $\left(\mathrm{D}=0.85 \times 10^{-10} \mathrm{~m}^{2} \mathrm{~s}^{-1}\right)$ and high constants $\left(\mathrm{D}=4.25 \times 10^{-10} \mathrm{~m}^{2} \mathrm{~s}^{-1}\right)$ are evident and may be related to the diffusions of unimolecular copolymers and LSR, respectively. Presumably, the trace with the highest constant $\left(\mathrm{D}=4.25 \times 10^{-10} \mathrm{~m}^{2} \mathrm{~s}^{-1}\right)$ suggests a rather high exchange between the free and the micelle-bound state of LSR. 


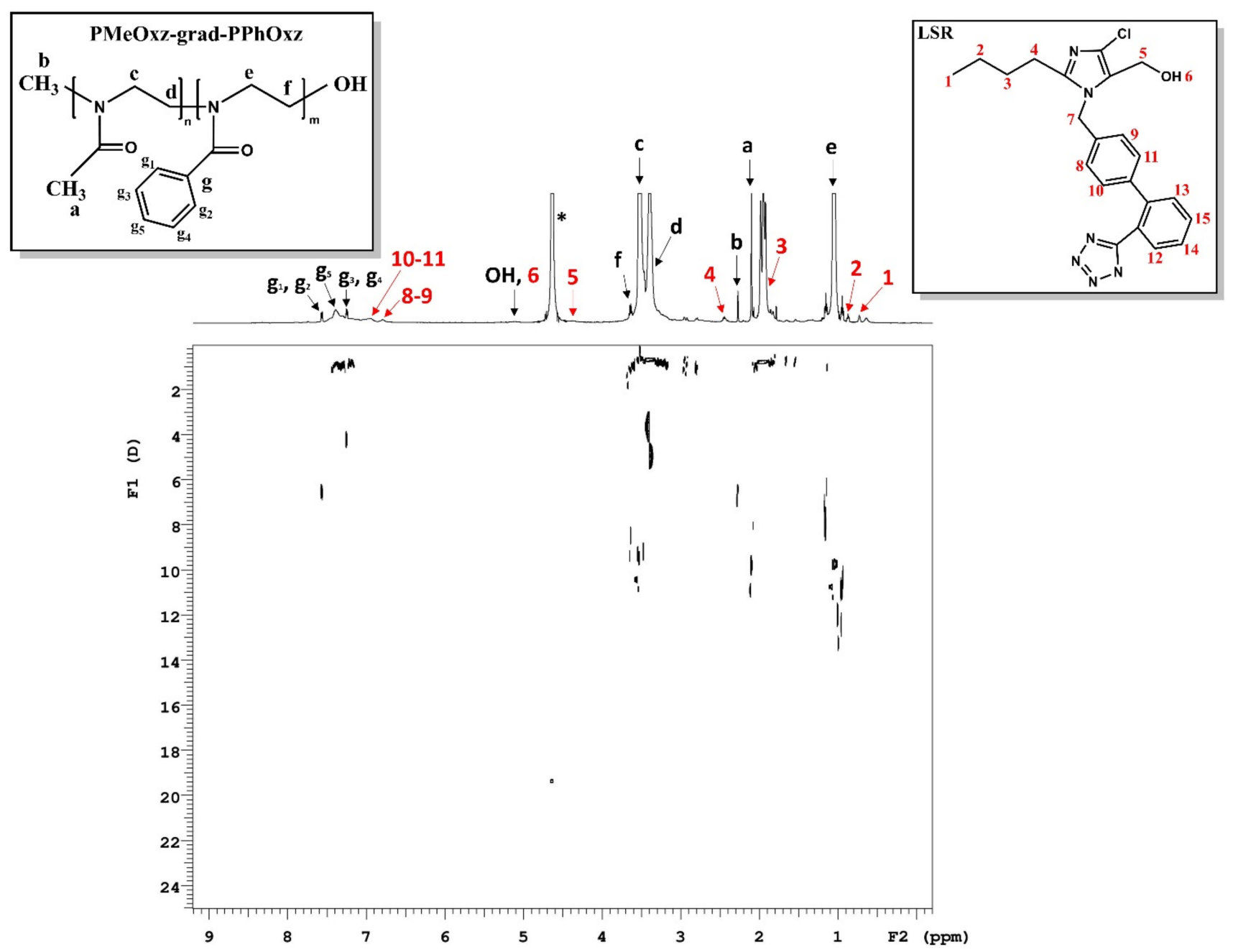

Figure 10. 2D-DOSY spectrum of PMeOxz72-grad-PPhOxz 28 + 50\% LSR nanocarriers. The black letters (a-g) denote the protons of the copolymer structure, the red letters point to the LSR peaks and the symbol * refers to the solvent $\mathrm{D}_{2} \mathrm{O}$.

\section{7. ${ }^{1} \mathrm{H}-\mathrm{NMR}$ Temperature Dependence Studies on PMeOxz $z_{22}$-Grad-PPhOxz 28 GC}

Additional ${ }^{1} \mathrm{H}-\mathrm{NMR}$ studies over a wide range of temperatures were conducted to reveal the mobility of protons during micellization. The ${ }^{1} \mathrm{H}-\mathrm{NMR}$ spectrum of $\mathrm{PMeOxz}_{72}$ grad-PPhOxz 28 GC at three different temperatures is displayed in Figure 11. The ${ }^{1} \mathrm{H}-\mathrm{NMR}$ peaks of $\mathrm{PMeOxz}_{72}$-grad-PPhOxz 28 GC presented in Figure 11, are shifting to lower field regions with increasing temperature. The half-width of $\mathrm{PMeOxz}_{72}$-grad-PPhOxz 28 proton signals is decreased, while their integrated intensity gradually increases from $25^{\circ} \mathrm{C}$ to $80^{\circ} \mathrm{C}$, implying the enhanced mobility of the protons.

The temperature-independent $\mathrm{PMeOxz}_{72}$-grad-PPhOxz 28 pseudo-diblock copolymer contains a not entirely hydrophobic core, due to the presence of hydrophilic MeOxz segments integrated in the core domain. Thus, heating may cause an increased fluidity of the core domain, inducing water penetration in the PhOxz-rich domain and further increase of proton mobility. 


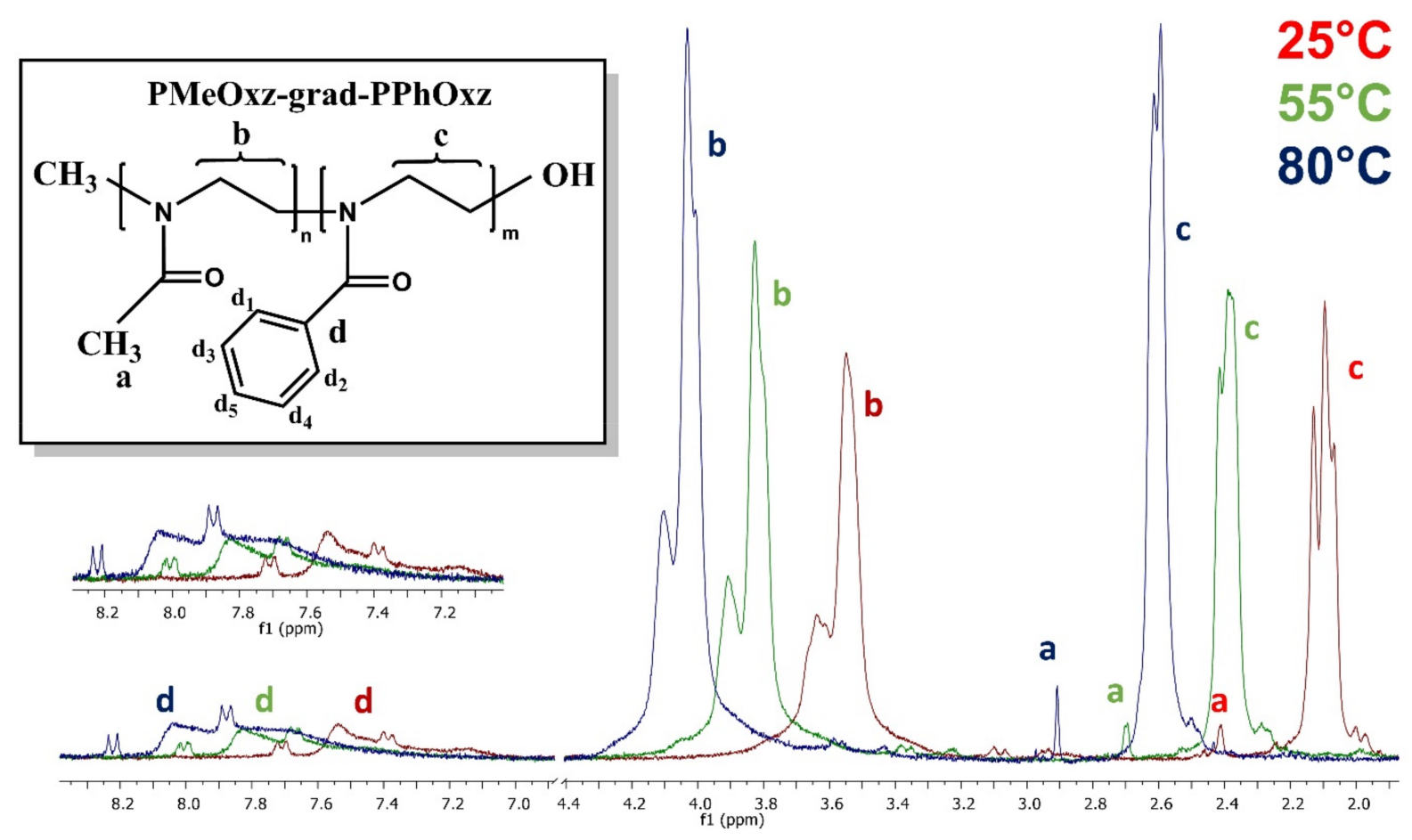

Figure 11. Comparison of ${ }^{1} \mathrm{H}-\mathrm{NMR}$ spectra of $\mathrm{PMeOxz}_{72}$-grad-PPhOxz 28 GC at various temperatures. The red, green, and blue letters refer to the protons of $\mathrm{PMeOxz}_{72}$-grad-PPhOxz 28 GC at $25^{\circ} \mathrm{C}, 55^{\circ} \mathrm{C}$ and $80{ }^{\circ} \mathrm{C}$, respectively.

\subsection{Encapsulation and Ultrasound Release Studies}

The non-invasive nature, safety and low cost of ultrasound-mediated release experiments conducted on polymeric nanocarriers contribute to controlled delivery of therapeutics, while in parallel reducing the unwanted side effects of traditional therapies [64-66]. Specifically, ultrasound penetrates through a vast number of different tissues triggering both mechanical and thermal phenomena. Additionally, ultrasound enhances drug release, visualizing the target during the therapeutic process [33]. Consequently, the ultrasoundtriggered release behavior of LSR from $\mathrm{PMeOxz}_{72}$-grad-PPhOxz 28 nanoassemblies was studied by means of UV-Vis spectroscopy.

The stable in serum solution of $\mathrm{PMeOxz}_{72}$-grad-PPhOxz 28 +50\% LSR nanocarriers was handpicked for the investigation of release kinetics, mainly due to the increased concentration of hydrophobic LSR (50\%). The \%DL and \%EE indexes were calculated using Equations (1) and (2) and are summarized in Table 6. The relatively high value of \%EE underlies an efficient loading of LSR into the gradient core of $\mathrm{PMeOxz}_{72}$-grad-PPhOxz 28 . Possibly, the similarity in the structural characteristics of $\mathrm{PMeOxz}_{72}$-grad-PPhOxz 28 and LSR (in terms of phenyl groups), as well as the GC structure and copolymer composition, favors the incorporation of amphiphilic LSR into the structure of nanoassemblies.

Table 6. Drug encapsulation and release characteristics of LSR loaded in PMeOxz 72 -grad-PPhOxz 28 nanoassemblies in water.

\begin{tabular}{cccc}
\hline Sample & \%DL & \%EE & \%Maximum Release Amount of LSR \\
\hline $\begin{array}{c}\text { PMeOxz72-grad-PPhOxz } 28 \\
\text { 50\% LSR nanocarriers }\end{array}$ & 12 & 87 & 45 \\
\hline
\end{tabular}

The cumulative drug release percentage vs. time is provided in Figure 12 for the $\mathrm{PMeOxz}_{72}$-grad-PPhOxz 28 + 50\% LSR nanocarriers. Based on UV-Vis results of Figure 12, an initial burst release of LSR is observed at the first fifteen minutes, followed by a continuous slow release of LSR, within five hours, without reaching a plateau. The initial 
burst release of LSR may be attributed to a small amount of the drug incorporated within hydrophobic-hydrophilic domains at the interface of the micelles, whereas the continuous slow release may be related to a larger amount of the drug encapsulated into the hydrophobic PPhOxz domains. The relatively low delayed LSR release (Table 6) implies that a significant portion of the encapsulated LSR is tightly bound to the PMeOxz72-grad$\mathrm{PPhOxz}_{28}$ nanoassemblies.

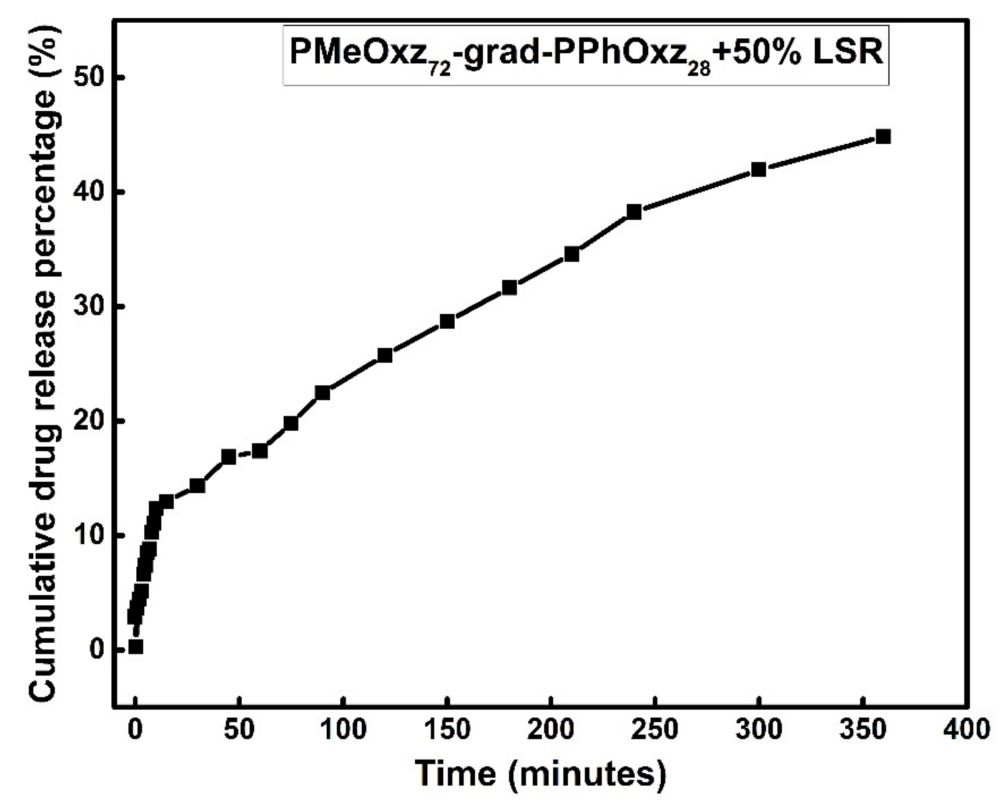

Figure 12. Release of LSR from $\mathrm{PMeOxz}_{72}$-grad-PPhOxz 28 nanoassemblies in aqueous media under sonication.

\section{Conclusions}

Highly stable drug-loaded biocompatible nanocarriers were formed by encapsulation of LSR into an amphiphilic PMeOxz 72 -grad-PPhOxz 28 GC through TFHM. A wide range of techniques was applied to study the physicochemical properties of the synthesized nanocarriers.

The physicochemical behavior of the nanoassemblies, as well as their drug encapsulation and drug release properties, were found to depend on the gradient molecular structure of the GC. Specifically, the presence of hydrophobic LSR molecules in the GC altered the structural characteristics of the GC/drug mixed assemblies by modulating the organization of the polymeric components.

Moreover, FBS interactions with $\mathrm{PMeOxz}_{72}$-grad-PPhOxz 28 GC and LSR-loaded $\mathrm{PMeOxz}_{72}$-grad-PPhOxz 28 nanocarriers manifested a good stability of the nanoassemblies and GC/drug mixed assemblies into a simulated biological environment, endorsing the idea that PMeOxz hydrophilic chains shield the GC nanoassemblies against protein adsorption and agglomeration. According to DLS stability studies, the LSR-loaded PMeOxz $72^{-}$ grad-PPhOxz 28 nanocarriers exhibited substantial long-term physical stability over time suggesting highly stable GC/drug mixed systems for a period of 23 days.

${ }^{1} \mathrm{H}-\mathrm{NMR}$ measurements further verified the existence of LSR in PMeOxz 72 -gradPPhOxz 28 nanoassemblies in $\mathrm{D}_{2} \mathrm{O}$ solutions. 2D-COSY, 2D-NOESY, and 2D-DOSY experiments confirmed the internal structure of the $\mathrm{PMeOxz}_{72}$-grad-PPhOxz 28 GC and LSR moieties, and supported the successful encapsulation of LSR into the micelles. The presence of hydrophilic PMeOxz segments in the gradient core of nanoassemblies induces an increased fluidity of the core matrix, especially upon heating, thus causing water penetration, resulting in increased proton mobility.

The similarity in the chemical structures of PMeOxz 72 -grad-PPhOxz 28 and LSR (in terms of phenyl groups), as well as the GC structure and copolymer composition, con- 
tributed to a significant value of \%EE (87\%) for LSR into the nanoassemblies. The relatively low LSR release (45\%) implied that a significant amount of the encapsulated LSR is strongly attached to the hydrophobic domains of $\mathrm{PMeOxz}_{72}$-grad-PPhOxz 28 nanoassemblies.

The current study presents an overall physicochemical evaluation of an amphiphilic GC and biocompatible drug-loaded nanocarriers, including NMR measurements. The aggregation behavior of the nanoassemblies is drastically regulated by the presence of hydrophobic drug molecules, while GC/drug intermolecular interactions are clearly evident in the system.

Author Contributions: Conceptualization, A.C., T.M. and S.P.; investigation, A.C. and T.M.; resources, S.P. and T.M.; writing—original draft preparation, A.C., T.M. and S.P.; writing-review and editing, A.C., T.M. and S.P.; supervision, S.P. and T.M. All authors have read and agreed to the published version of the manuscript.

Funding: This research received no external funding.

Institutional Review Board Statement: Not applicable.

Informed Consent Statement: Not applicable.

Data Availability Statement: The data presented in this study are available on request from the corresponding author.

Acknowledgments: Materials were supported by National Kapodistrian University of Athens (NKUA). NMR experiments were supported by CERIC 20187056.

Conflicts of Interest: The authors have no competing interest to declare.

\section{References}

1. Kuperkar, K.; Tiwari, S.; Bahadur, P. Chapter 15-Self-Assembled Block Copolymer Nanoaggregates for Drug Delivery Applications. In Applications of Polymers in Drug Delivery, 2nd ed.; Misra, A., Shahiwala, A., Eds.; Elsevier: Cambridge, MA, USA, 2021; pp. 423-447. [CrossRef]

2. Avramović, N.; Mandić, B.; Savić-Radojević, A.; Simić, T. Polymeric nanocarriers of drug delivery systems in cancer therapy. Pharmaceutics 2020, 12, 298. [CrossRef] [PubMed]

3. Kwon, G.S.; Kataoka, K. Block copolymer micelles as long-circulating drug vehicles. Adv. Drug Deliver. Rev. 2012, 64, 237-245. [CrossRef]

4. Raveendran, R.; Mullen, K.M.; Wellard, R.M.; Sharma, C.P.; Hoogenboom, R.; Dargaville, T.R. Poly (2-oxazoline) block copolymer nanoparticles for curcumin loading and delivery to cancer cells. Eur. Polym. J. 2017, 93, 682-694. [CrossRef]

5. Patra, J.K.; Das, G.; Fraceto, L.F.; Campos, E.V.R.; Rodriguez-Torres, M.D.P.; Acosta-Torres, L.S.; Diaz-Torres, L.A.; Grillo, R.; Swamy, M.K.; Sharma, S.; et al. Nano based drug delivery systems: Recent developments and future prospects. J. Nanobiotechnol. 2018, 16, 71. [CrossRef]

6. Chen, Y.; Chen, H.; Feng, M.; Dong, Y. Amphiphilic gradient copolymers: Synthesis, self-assembly, and applications. Eur. Polym. J. 2016, 85, 489-498. [CrossRef]

7. Filippov, S.K.; Verbraeken, B.; Konarev, P.V.; Svergun, D.I.; Angelov, B.; Vishnevetskaya, N.S.; Papadakis, C.M.; Rogers, S.; Radulescu, A.; Courtin, T.; et al. Block and Gradient Copoly(2-oxazoline) Micelles: Strikingly Different on the Inside. J. Phys. Chem. Lett. 2017, 8, 3800-3804. [CrossRef]

8. Oleszko-Torbus, N.; Utrata-Wesołek, A.; Wałach, W.; Dworak, A. Solution behavior of thermoresponsive random and gradient copolymers of 2-n-propyl-2-oxazoline. Eur. Polym. J. 2017, 88, 613-622. [CrossRef]

9. Černochová, Z.; Bogomolova, A.; Borisova, O.V.; Filippov, S.K.; Černoch, P.; Billon, L.; Borisov, O.V.; Štěpánek, P. Thermodynamics of the multi-stage self-assembly of pH-sensitive gradient copolymers in aqueous solutions. Soft Matter 2016, 12, 6788-6798. [CrossRef]

10. Rabyk, M.; Destephen, A.l.; Lapp, A.; King, S.; Noirez, L.; Billon, L.; Hruby, M.; Borisov, O.; Stepanek, P.; Deniau, E. Interplay of thermosensitivity and $\mathrm{pH}$ sensitivity of amphiphilic block-gradient copolymers of dimethylaminoethyl acrylate and styrene. Macromolecules 2018, 51, 5219-5233. [CrossRef]

11. Borisova, O.; Billon, L.; Zaremski, M.; Grassl, B.; Bakaeva, Z.; Lapp, A.; Stepanek, P.; Borisov, O. Synthesis and pH-and salinity-controlled self-assembly of novel amphiphilic block-gradient copolymers of styrene and acrylic acid. Soft Matter 2012, 8, 7649-7659. [CrossRef]

12. Zhang, Q.; Vancoillie, G.; Mees, M.A.; Hoogenboom, R. Thermoresponsive polymeric temperature sensors with broad sensing regimes. Polym. Chem. 2015, 6, 2396-2400. [CrossRef]

13. Chen, J.; Li, B.; Li, X.; Zhang, J.; Wan, X. Gradient helical copolymers: Synthesis, chiroptical properties, thermotropic liquid crystallinity, and self-assembly in selective organic solvents. Polym. Chem. 2018, 9, 2002-2010. [CrossRef] 
14. Zhu, C.; Yao, R.; Chen, Y.; Feng, M.; Ma, S.; Zhang, C. Self-assembly of fluorinated gradient copolymer in three-dimensional co-flow focusing microfluidic. J. Colloid Interface Sci. 2018, 526, 75-82. [CrossRef]

15. Eggers, S.; Eckert, T.; Abetz, V. Double thermoresponsive block-random copolymers with adjustable phase transition temperatures: From block-like to gradient-like behavior. J. Polym. Sci. Part A Polym. Chem. 2018, 56, 399-411. [CrossRef]

16. Yañez-Macias, R.; Kulai, I.; Ulbrich, J.; Yildirim, T.; Sungur, P.; Hoeppener, S.; Guerrero-Santos, R.; Schubert, U.S.; Destarac, M.; Guerrero-Sanchez, C. Thermosensitive spontaneous gradient copolymers with block-and gradient-like features. Polym. Chem. 2017, 8, 5023-5032. [CrossRef]

17. Lorson, T.; Lubtow, M.M.; Wegener, E.; Haider, M.S.; Borova, S.; Nahm, D.; Jordan, R.; Sokolski-Papkov, M.; Kabanov, A.V.; Luxenhofer, R. Poly(2-oxazoline)s based biomaterials: A comprehensive and critical update. Biomaterials 2018, 178, 204-280. [CrossRef]

18. Zheng, C. Gradient copolymer micelles: An introduction to structures as well as structural transitions. Soft Matter 2019, 15, 5357-5370. [CrossRef]

19. Kravchenko, V.S.; Potemkin, I.I. Micelles of Gradient vs Diblock Copolymers: Difference in the Internal Structure and Properties. J. Phys. Chem. B 2016, 120,12211-12217. [CrossRef]

20. Steinhauer, W.; Hoogenboom, R.; Keul, H.; Moeller, M. Block and gradient copolymers of 2-hydroxyethyl acrylate and 2methoxyethyl acrylate via RAFT: Polymerization kinetics, thermoresponsive properties, and micellization. Macromolecules 2013, 46, 1447-1460. [CrossRef]

21. Ribaut, T.; Oberdisse, J.; Annighofer, B.; Fournel, B.; Sarrade, S.; Haller, H.; Lacroix-Desmazes, P. Solubility and self-assembly of amphiphilic gradient and block copolymers in supercritical $\mathrm{CO}_{2}$. J. Phys. Chem. B 2011, 115, 836-843. [CrossRef]

22. Pandav, G.; Pryamitsyn, V.; Gallow, K.C.; Loo, Y.-L.; Genzer, J.; Ganesan, V. Phase behavior of gradient copolymer solutions: A Monte Carlo simulation study. Soft Matter 2012, 8, 6471-6482. [CrossRef]

23. Kuldová, J.; Košovan, P.; Limpouchová, Z.; Procházka, K. Computer Study of the Association Behavior of Gradient Copolymers: Analysis of Simulation Results Based on a New Algorithm for Recognition and Classification of Aggregates. Macromol. Theory Simul. 2013, 22, 61-70. [CrossRef]

24. Bonné, T.B.; Lüdtke, K.; Jordan, R.; Papadakis, C.M. Effect of polymer architecture of amphiphilic poly (2-oxazoline) copolymers on the aggregation and aggregate structure. Macromol. Chem. Phys. 2007, 208, 1402-1408. [CrossRef]

25. Luxenhofer, R.; Han, Y.; Schulz, A.; Tong, J.; He, Z.; Kabanov, A.V.; Jordan, R. Poly (2-oxazoline) s as Polymer Therapeutics. Macromol. Rapid Commun. 2012, 33, 1613-1631. [CrossRef]

26. Verbrugghen, T.; Monnery, B.D.; Glassner, M.; Stroobants, S.; Hoogenboom, R.; Staelens, S. $\mu$ PET imaging of the pharmacokinetic behavior of medium and high molar mass 89Zr-labeled poly (2-ethyl-2-oxazoline) in comparison to poly (ethylene glycol). J. Control. Release 2016, 235, 63-71. [CrossRef]

27. Kronek, J.; Kronekova, Z.; Luston, J.; Paulovicova, E.; Paulovicova, L.; Mendrek, B. In vitro bio-immunological and cytotoxicity studies of poly(2-oxazolines). J. Mater. Sci. Mater. Med. 2011, 22, 1725-1734. [CrossRef]

28. Luxenhofer, R.; Sahay, G.; Schulz, A.; Alakhova, D.; Bronich, T.K.; Jordan, R.; Kabanov, A.V. Structure-property relationship in cytotoxicity and cell uptake of poly(2-oxazoline) amphiphiles. J. Control. Release 2011, 153, 73-82. [CrossRef]

29. He, Z.; Wan, X.; Schulz, A.; Bludau, H.; Dobrovolskaia, M.A.; Stern, S.T.; Montgomery, S.A.; Yuan, H.; Li, Z.; Alakhova, D.; et al. A high capacity polymeric micelle of paclitaxel: Implication of high dose drug therapy to safety and in vivo anti-cancer activity. Biomaterials 2016, 101, 296-309. [CrossRef]

30. Milonaki, Y.; Kaditi, E.; Pispas, S.; Demetzos, C. Amphiphilic gradient copolymers of 2-methyl-and 2-phenyl-2-oxazoline: Self-organization in aqueous media and drug encapsulation. J. Polym. Sci. Part A Polym. Chem. 2012, 50, 1226-1237. [CrossRef]

31. Luxenhofer, R.; Schulz, A.; Roques, C.; Li, S.; Bronich, T.K.; Batrakova, E.V.; Jordan, R.; Kabanov, A.V. Doubly amphiphilic poly (2-oxazoline) s as high-capacity delivery systems for hydrophobic drugs. Biomaterials 2010, 31, 4972-4979. [CrossRef]

32. Qiu, L.-Y.; Yan, L.; Zhang, L.; Jin, Y.-M.; Zhao, Q.-H. Folate-modified poly (2-ethyl-2-oxazoline) as hydrophilic corona in polymeric micelles for enhanced intracellular doxorubicin delivery. Int. J. Pharm. 2013, 456, 315-324. [CrossRef] [PubMed]

33. Salgarella, A.R.; Zahoranová, A.; Šrámková, P.; Majerčíková, M.; Pavlova, E.; Luxenhofer, R.; Kronek, J.; Lacík, I.; Ricotti, L. Investigation of drug release modulation from poly (2-oxazoline) micelles through ultrasound. Sci. Rep. 2018, 8, 1-13. [CrossRef] [PubMed]

34. Zhang, P.; Qian, X.; Zhang, Z.; Li, C.; Xie, C.; Wu, W.; Jiang, X. Supramolecular Amphiphilic Polymer-Based Micelles with Seven-Armed Polyoxazoline Coating for Drug Delivery. ACS Appl. Mater. Interfaces 2017, 9, 5768-5777. [CrossRef] [PubMed]

35. Wan, X.; Min, Y.; Bludau, H.; Keith, A.; Sheiko, S.S.; Jordan, R.; Wang, A.Z.; Sokolsky-Papkov, M.; Kabanov, A.V. Drug Combination Synergy in Worm-like Polymeric Micelles Improves Treatment Outcome for Small Cell and Non-Small Cell Lung Cancer. ACS Nano 2018, 12, 2426-2439. [CrossRef]

36. Claeys, B.; Vervaeck, A.; Vervaet, C.; Remon, J.P.; Hoogenboom, R.; De Geest, B.G. Poly(2-ethyl-2-oxazoline) as matrix excipient for drug formulation by hot melt extrusion and injection molding. Macromol. Rapid Commun. 2012, 33, 1701-1707. [CrossRef]

37. Boel, E.; Smeets, A.; Vergaelen, M.; De la Rosa, V.R.; Hoogenboom, R.; Van den Mooter, G. Comparative study of the potential of poly(2-ethyl-2-oxazoline) as carrier in the formulation of amorphous solid dispersions of poorly soluble drugs. Eur. J. Pharm. Biopharm. 2019, 144, 79-90. [CrossRef] 
38. Everaerts, M.; Tigrine, A.; de la Rosa, V.R.; Hoogenboom, R.; Adriaensens, P.; Clasen, C.; Van den Mooter, G. Unravelling the Miscibility of Poly(2-oxazoline)s: A Novel Polymer Class for the Formulation of Amorphous Solid Dispersions. Molecules 2020, 25, 3587. [CrossRef]

39. Mavromoustakos, T.; Zervou, M.; Zoumpoulakis, P.; Kyrikou, I.; Benetis, N.P.; Polevaya, L.; Roumelioti, P.; Giatas, N.; Zoga, A.; Minakakis, P.M.; et al. Conformation and bioactivity. Design and discovery of novel antihypertensive drugs. Curr. Top. Med. Chem. 2004, 4, 385-401. [CrossRef]

40. Peng, F.; Li, R.; Zhang, F.; Qin, L.; Ling, G.; Zhang, P. Potential drug delivery nanosystems for improving tumor penetration. Eur. J. Pharm. Biopharm. 2020, 151, 220-238. [CrossRef]

41. Zervou, M.; Cournia, Z.; Potamitis, C.; Patargias, G.; Durdagi, S.; Grdadolnik, S.G.; Mavromoustakos, T. Insights into the molecular basis of action of the AT1 antagonist losartan using a combined NMR spectroscopy and computational approach. Biochim. Biophys. Acta 2014, 1838, 1031-1046. [CrossRef]

42. Hoogenboom, R.; Wiesbrock, F.; Huang, H.; Leenen, M.A.M.; Thijs, H.M.L.; van Nispen, S.F.G.M.; van der Loop, M.; Fustin, C.-A.; Jonas, A.M.; Gohy, J.-F.; et al. Microwave-Assisted Cationic Ring-Opening Polymerization of 2-Oxazolines: A Powerful Method for the Synthesis of Amphiphilic Triblock Copolymers. Macromolecules 2006, 39, 4719-4725. [CrossRef]

43. Hamada, T.; Kudo, K. Nanoaggregate Formation of Amphiphilic Alternating and Random Copolyimides in Water. Chem. Lett. 2010, 39, 1285-1287. [CrossRef]

44. Lambermont-Thijs, H.M.; Heuts, J.P.; Hoeppener, S.; Hoogenboom, R.; Schubert, U.S. Selective partial hydrolysis of amphiphilic copoly (2-oxazoline) s as basis for temperature and $\mathrm{pH}$ responsive micelles. Polym. Chem. 2011, 2, 313-322. [CrossRef]

45. Chroni, A.; Mavromoustakos, T.; Pispas, S. Biocompatible PEO-b-PCL Nanosized Micelles as Drug Carriers: Structure and Drug-Polymer Interactions. Nanomaterials 2020, 10, 1872. [CrossRef]

46. Chroni, A.; Mavromoustakos, T.; Pispas, S. Nano-Assemblies from Amphiphilic PnBA-b-POEGA Copolymers as Drug Nanocarriers. Polymers 2021, 13, 1164. [CrossRef]

47. Hadjichristidis, N.; Pispas, S.; Floudas, G. Block Copolymers: Synthetic Strategies, Physical Properties, and Applications; John Wiley \& Sons: New York, NY, USA, 2003.

48. Hodrokoukes, P.; Pispas, S.; Hadjichristidis, N. Controlling micellar properties of styrene/isoprene copolymers by altering the monomer arrangement along the chain. Macromolecules 2002, 35, 834-840. [CrossRef]

49. Hoogenboom, R.; Thijs, H.M.; Wouters, D.; Hoeppener, S.; Schubert, U.S. Tuning solution polymer properties by binary water-ethanol solvent mixtures. Soft Matter 2008, 4, 103-107. [CrossRef]

50. Hoogenboom, R.; Thijs, H.M.; Wouters, D.; Hoeppener, S.; Schubert, U.S. Solvent responsive micelles based on block and gradient copoly (2-oxazoline) s. Macromolecules 2008, 41, 1581-1583. [CrossRef]

51. Bronstein, L.M.; Chernyshov, D.M.; Timofeeva, G.I.; Dubrovina, L.V.; Valetsky, P.M.; Obolonkova, E.S.; Khokhlov, A.R. Interaction of polystyrene-block-poly (ethylene oxide) micelles with cationic surfactant in aqueous solutions. Metal colloid formation in hybrid systems. Langmuir 2000, 16, 3626-3632. [CrossRef]

52. Pispas, S. Vesicular structures in mixed block copolymer/surfactant solutions. Soft Matter 2011, 7, 8697-8701. [CrossRef]

53. Pispas, S. Self-assembled nanostructures in mixed anionic-neutral double hydrophilic block copolymer/cationic vesicle-forming surfactant solutions. Soft Matter 2011, 7, 474-482. [CrossRef]

54. Burke, S.E.; Eisenberg, A. Effect of Sodium Dodecyl sulfate on the morphology of polystyrene-b-poly (acrylic acid) aggregates in dioxane- water Mixtures. Langmuir 2001, 17, 8341-8347. [CrossRef]

55. Satyanarayana, D.; Kannan, K.; Manavalan, R. Artifitial neural network calibration models for simultaneous spectrophotometric determination of atenolol and losartan potassium in tablets. Chem. Anal. 2006, 51, 771.

56. Pawar, H.A.; Lalitha, K.G. Development and Validation of a Novel RP-HPLC Method for Estimation of Losartan Potassium in Dissolution Samples of Immediate and Sustained Release Tablets. Chromatogr. Res. Int. 2014, 2014, 1-8. [CrossRef]

57. Maggio, R.M.; Castellano, P.M.; Kaufman, T.S. A multivariate approach for the simultaneous determination of losartan potassium and hydrochlorothiazide in a combined pharmaceutical tablet formulation. Anal. Bioanal. Chem. 2008, 391, 2949-2955. [CrossRef] [PubMed]

58. Al-Majed, A.-R.A.; Assiri, E.; Khalil, N.Y.; Abdel-Aziz, H.A. Losartan: Comprehensive profile. In Profiles of Drug Substances, Excipients and Related Methodology; Elsevier: Cambridge, MA, USA, 2015; Volume 40, pp. 159-194. [CrossRef]

59. Orozco, S.; Blanco, L.; Garza, M.; González, V.; Borrás, C.; Sharifker, B. Phenol degradation using glassy carbon electrodes modified with particles of Co-Mo alloy. Int. J. Electrochem. Sci. 2013, 8, 5698-5709.

60. Pippa, N.; Kaditi, E.; Pispas, S.; Demetzos, C. PEO-b-PCL-DPPC chimeric nanocarriers: Self-assembly aspects in aqueous and biological media and drug incorporation. Soft Matter 2013, 9, 4073. [CrossRef]

61. Palchetti, S.; Colapicchioni, V.; Digiacomo, L.; Caracciolo, G.; Pozzi, D.; Capriotti, A.L.; La Barbera, G.; Lagana, A. The protein corona of circulating PEGylated liposomes. Biochim. Biophys. Acta 2016, 1858, 189-196. [CrossRef]

62. Deyerle, B.A.; Zhang, Y. Effects of Hofmeister anions on the aggregation behavior of PEO-PPO-PEO triblock copolymers. Langmuir 2011, 27, 9203-9210. [CrossRef]

63. Patel, K.; Bharatiya, B.; Kadam, Y.; Bahadur, P. Micellization and clouding behavior of EO-PO block copolymer in aqueous salt solutions. J. Surfactants Deterg. 2010, 13, 89-95. [CrossRef]

64. Sirsi, S.R.; Borden, M.A. State-of-the-art materials for ultrasound-triggered drug delivery. Adv. Drug Deliv. Rev. 2014, 72, 3-14. [CrossRef] 
65. Rapoport, N. Ultrasound-mediated micellar drug delivery. Int. J. Hyperth. 2012, 28, 374-385. [CrossRef]

66. Xia, H.; Zhao, Y.; Tong, R. Ultrasound-Mediated Polymeric Micelle Drug Delivery. Adv. Exp. Med. Biol. 2016, 880, 365-384. [CrossRef] 